\title{
A high-order scheme to approximate the Caputo fractional derivative and its application to solve the fractional diffusion wave equation
}

\author{
Ruilian $\mathrm{Du}^{\mathrm{a}}$, Yubin Yan ${ }^{\mathrm{b}}$, Zongqi Liang*,a \\ ${ }^{a}$ School of Science, Jimei University, Xiamen, Fujian 361021, P. R. China \\ ${ }^{b}$ Department of Mathematics, University of Chester, CH1 4BJ, UK
}

\begin{abstract}
A new high-order finite difference scheme to approximate the Caputo fractional derivative $\frac{1}{2}\left({ }_{0}^{C} D_{t}^{\alpha} f\left(t_{k}\right)+\right.$ $\left.{ }_{0}^{C} D_{t}^{\alpha} f\left(t_{k-1}\right)\right), k=1,2, \ldots, N$, with the convergence order $O\left(\Delta t^{4-\alpha}\right), \alpha \in(1,2)$ is obtained when $f^{\prime \prime \prime}\left(t_{0}\right)=0$, where $\Delta t$ denotes the time step size. Based on this scheme we introduce a finite difference method for solving fractional diffusion wave equation with the convergence order $O\left(\Delta t^{4-\alpha}+h^{2}\right)$, where $h$ denotes the space step size. Numerical examples are given to show that the numerical results are consistent with the theoretical results.
\end{abstract}

Key words:

Finite difference method, fractional diffusion wave equation, Caputo fractional derivative

AMS Subject Classification: 65M12; 65M06; 65M70;35S10

\section{Introduction}

In this paper, we introduce a new high-order finite difference scheme to approximate $\frac{1}{2}\left({ }_{0}^{C} D_{t}^{\alpha} f\left(t_{k}\right)+\right.$ $\left.{ }_{0}^{C} D_{t}^{\alpha} f\left(t_{k-1}\right)\right), k=1,2, \ldots, N$, where the Caputo fractional derivative ${ }_{0}^{C} D_{t}^{\alpha} f(t), 1<\alpha<2$ is defined by

$$
{ }_{0}^{C} D_{t}^{\alpha} f(t)=\frac{1}{\Gamma(2-\alpha)} \int_{0}^{t}(t-\tau)^{1-\alpha} f^{\prime \prime}(\tau) d \tau,
$$

and $f^{\prime \prime}(\tau)$ denotes the second order derivative of $f$. Based on this scheme, we construct a new finite difference method for solving the following fractional diffusion wave equation, with $1<\alpha<2$,

$$
\begin{aligned}
& { }_{0}^{C} D_{t}^{\alpha} u(x, t)=\frac{\partial^{2} u(x, t)}{\partial x^{2}}+F(x, t), \quad x \in(0,1), t \in(0, T], \\
& u(x, 0)=u_{0}(x), \quad u_{t}(x, 0)=u_{1}(x), \\
& u(0, t)=\phi(t), \quad u(1, t)=\varphi(t), t \in(0, T],
\end{aligned}
$$

where $F(x, t), u_{0}(x), u_{1}(x), \phi(t)$ and $\varphi(t)$ denote the source term, boundary conditions and the initial conditions, respectively.

The system (2)-(4) can be used to model many physical problems, see [25], [22], [26] and the references therein. The theoretical analysis can be found, for example, in [1], [2], [9], [10], [11], [20], [21].

\footnotetext{
*Corresponding author. This work is funded partially by the following: the Science and Technology Department key projects of Fujian Province, China (grant 2014H0034); the key project for Industry-Academia-Research of Fujian Province, China(grant 2017H6015); the Natural Science Foundation of Fujian Province, China (grant 2017J01402, 2017J01557, 2016J01310, 2016J01309) and Department of Education, University Fund key projects of Fujian Province, China (grant J2160450).

Email addresses: drl158@163.com (Ruilian Du), y.yan@chester.ac.uk (Yubin Yan), zqliang@jmu.edu.cn (Zongqi Liang ) 
In general, it is not possible to obtain the analytic solution of the system (2)-(4). Therefore we need to design some numerical methods for solving (2)-(4). Since the fractional derivative is a nonlocal operator, the fractional derivative of $f$ at time $t$ depends on all the previous values of $f(s)$ for $0<s<t$. Compared with the discretization of the integer-order derivative, the computation of the fractional derivative needs more time and requires more computer memory to store the data. In recent years, designing some efficient high-order numerical methods for solving fractional related differential equation is a very active research topic.

Let us review some schemes to approximate the Caputo fractional derivative ${ }_{0}^{C} D_{t}^{\alpha} f(t), 1<\alpha<2$ in literature. Let $0=t_{0}<t_{1}<\cdots<t_{N}=T$ be a time partition on $[0, T]$ and let $\Delta t$ denote the time step size. One way to approximate the Caputo fractional derivative ${ }_{0}^{C} D_{t}^{\alpha} f\left(t_{k}\right), k=1,2, \ldots, N$ with $1<\alpha<2$ is by using the direct method. More precisely, we write the Caputo fractional derivative (1) as

$$
{ }_{0}^{C} D_{t}^{\alpha} f\left(t_{k}\right)=\frac{1}{\Gamma(2-\alpha)} \sum_{j=1}^{k} \int_{t_{j-1}}^{t_{j}} \frac{f^{\prime \prime}(\tau) d \tau}{\left(t_{k}-\tau\right)^{\alpha-1}} .
$$

On $\left[t_{j-1}, t_{j}\right]$, we approximate $f^{\prime \prime}(\tau)$ by $\frac{f\left(t_{j-1}\right)-2 f\left(t_{j}\right)+f\left(t_{j+1}\right)}{\Delta t^{2}}$ and obtain the following $L 2$ method, [24], [16],

$$
{ }_{0}^{C} D_{t}^{\alpha} f\left(t_{k}\right) \approx \frac{\Delta t^{-\alpha}}{\Gamma(3-\alpha)} \sum_{j=0}^{k-1} b_{j}\left[f\left(t_{k-j-1}\right)-2 f\left(t_{k-j}\right)+f\left(t_{k-j+1}\right)\right],
$$

where

$$
b_{j}=(j+1)^{2-\alpha}-j^{2-\alpha} .
$$

If we use the four-point discretization $\frac{\left(f\left(t_{j+1}\right)-f\left(t_{j}\right)\right)-\left(f\left(t_{j-1}\right)-f\left(t_{j-2}\right)\right)}{2 \Delta t^{2}}$ to approximate $f^{\prime \prime}(\tau)$ on $\left[t_{j-1}, t_{j}\right]$, we get the following $L 2 C$ method, [24], [16],

$$
{ }_{0}^{C} D_{t}^{\alpha} f\left(t_{k}\right) \approx \frac{\Delta t^{-\alpha}}{\Gamma(3-\alpha)} \sum_{j=0}^{k-1} b_{j}\left[\left(f\left(t_{k-j-2}\right)-f\left(t_{k-j-1}\right)\right)-\left(f\left(t_{k-j}\right)-f\left(t_{k-j+1}\right)\right)\right],
$$

where $f\left(t_{-1}\right)$ for $k=1$ can be considered as in [24]. Both $L 2$ and $L 2 C$ method has the convergence order $O\left(\Delta t^{3-\alpha}\right), 1<\alpha<2$.

Another way to approximate the Caputo fractional derivative ${ }_{0}^{C} D_{t}^{\alpha} f\left(t_{k}\right), k=1,2, \ldots, N$ with $1<\alpha<2$ is by using the relation between the Caputo fractional derivative and the Riemann-Liouville fractional derivative. More precisely, we use the following approximation scheme

$$
\begin{aligned}
{ }_{0}^{C} D_{t}^{\alpha} f\left(t_{k}\right) & =\left.{ }_{0}^{R} D_{t}^{\alpha}\left[f(t)-f(0)-\frac{f^{\prime}(0)}{1 !} t\right]\right|_{t=t_{k}} \\
& =-\frac{f(0) t_{k}^{-\alpha}}{\Gamma(1-\alpha)}-\frac{f^{\prime}(0) t_{k}^{1-\alpha}}{\Gamma(2-\alpha)}+D^{2}\left({ }_{0}^{R} D_{t}^{-(2-\alpha)} f\right)\left(t_{k}\right) \\
& \approx-\frac{f(0) t_{k}^{-\alpha}}{\Gamma(1-\alpha)}-\frac{f^{\prime}(0) t_{k}^{1-\alpha}}{\Gamma(2-\alpha)}+\frac{1}{\Delta t^{2}}\left({ }_{0}^{R} D_{t}^{-(2-\alpha)} f\left(t_{k+1}\right)-2_{0}^{R} D_{t}^{-(2-\alpha)} f\left(t_{k}\right)+{ }_{0}^{R} D_{t}^{-(2-\alpha)} f\left(t_{k-1}\right)\right)
\end{aligned}
$$

where ${ }_{0}^{R} D_{t}^{\beta} f(t)$ denotes the Riemann-Liouville fractional derivative $(\beta>0)$ or Riemann-Liouville fractional integral $(\beta<0)$ and $D^{2} v(t)=v^{\prime \prime}(t)$ denotes the second order derivative of $v(t)$. Here the second order derivative of $D^{-(2-\alpha)} f(t)$ at $t_{k}$ is approximated by using the central difference formula. Further if we use the piecewise linear interpolation polynomial to approximate the Riemann-Liouville integral ${ }_{0}^{R} D_{t}^{-(2-\alpha)} f(t)$ at $t_{l}, l=k-1, k, k+1$, then a second-order scheme to approximate the Caputo fractional derivative is obtained, see, e.g., [4], [27]. There are other approaches to approximate the Caputo fractional derivative, for example, by using the Lubich method [19], the weighted and shifted Grünwald-Letnikov difference (WSGD) [30], the 
Hadamard finite-integral method [8], etc. In this paper, we shall use the direct method to approximate the Caputo fractional derivative, see Section 2 below.

Let us now review some finite difference methods for solving (2)-(4). Sun and Wu [29] considered a finite difference method for solving (2)-(4) with the convergence order $O\left(\Delta t^{3-\alpha}+h^{2}\right)$ and the stability and error estimates are proved by using the energy method, where $h$ denotes the space step size. Du et al. [7] considered a finite difference method for solving (2)-(4) with the convergence order $O\left(\Delta t^{3-\alpha}+h^{4}\right)$ by using the compact finite difference scheme. By using the equivalent integro-differential form of (2)-(4), Huang et al. [13] introduced two finite difference methods for solving (2)-(4). Zeng [35] proposed some finite difference schemes of second-order accuracy in both time and space for solving (2)-(4) by using the fractional multistep methods developed by Lubich [19] in time and central difference formula in space. The stability and error estimates are proved by using the Fourier method. Other finite difference methods for solving (2)-(4) can be found in, e.g., , [5], [12], [31], [34], [4], [32], [6], etc.

In [28], Sun and Gao introduced a finite difference scheme to approximate the Caputo fractional derivative $\frac{1}{2}\left({ }_{0}^{C} D_{t}^{\alpha} f\left(t_{k}\right)+{ }_{0}^{C} D_{t}^{\alpha} f\left(t_{k-1}\right)\right)$ with the convergence order $O\left(\Delta t^{3-\alpha}\right), 1<\alpha<2$ and applied this scheme to solve the fractional diffusion wave equation (2)-(4). In this paper, we introduce a new high-order approximation scheme with the convergence order $O\left(\Delta t^{4-\alpha}\right), 1<\alpha<2$ when $f^{\prime \prime \prime}\left(t_{0}\right)=0$ to approximate the Caputo fractional derivative $\frac{1}{2}\left({ }_{0}^{C} D_{t}^{\alpha} f\left(t_{k}\right)+{ }_{0}^{C} D_{t}^{\alpha} f\left(t_{k-1}\right)\right)$ and apply this scheme to obtain a high-order numerical method for solving the fractional diffusion wave equation (2)-(4). The properties of the weights in the proposed approximation scheme are studied and the truncation errors of the proposed numerical methods for solving diffusion wave equation are derived. For the high-order schemes to approximate the Caputo fractional derivative ${ }_{0}^{C} D_{t}^{\alpha} f\left(t_{k}\right)$ with $0<\alpha<1$, we refer to [17], [3], [15], [14], [23], [33], [18] and the references therein. Since the approximation of the Caputo fractional derivative ${ }_{0}^{C} D_{t}^{\alpha} f\left(t_{k}\right)$ with $1<\alpha<2$ involves the approximation of the second order derivative $f^{\prime \prime}(t)$ which is more challenging than the approximation of the Caputo fractional derivative with $0<\alpha<1$ where one only need to approximate the first order derivative $f^{\prime}(t)$.

The paper is organized as follows. In Section 2, we consider the approximation of the Caputo fractional derivative and the truncation error of the approximation scheme is derived. In Section 3, we consider the finite difference method for solving the fractional diffusion wave equations and the properties of the weights in the approximation scheme to the Caputo fractional derivative are studied. Finally in Section 4, we give some numerical examples to show that the numerical results are consistent with the theoretical results.

In this paper, we denote $C$ as a positive constant independent of the functions and parameters concerned, but not necessarily the same at different occurrences.

\section{A high-order scheme to approximate the Caputo fractional derivative}

In this section, we will introduce an approximate scheme to Caputo fractional derivative ${ }_{0}^{C} D_{t}^{\alpha} f(t)$ at $t=t_{k}, k=2,3, \ldots, N$. Let $g(\tau)=f^{\prime}(\tau)$, then (6) can be written as, with $k=2,3, \ldots, N$,

$$
{ }_{0}^{C} D_{t}^{\alpha} f\left(t_{k}\right)=\frac{1}{\Gamma(2-\alpha)} \sum_{j=1}^{k} \int_{t_{j-1}}^{t_{j}} \frac{g^{\prime}(\tau) d \tau}{\left(t_{k}-\tau\right)^{\alpha-1}} .
$$

We shall use the linear interpolation polynomial $P_{1,1} g$ to approximate $g$ on the first small interval $\left[t_{0}, t_{1}\right]$ and the quadratic interpolation polynomial $P_{2, j} g$ to approximate $g$ on the interval $\left[t_{j-2}, t_{j}\right], j \geq 2$, where

$$
P_{1,1} g(t)=g\left(t_{0}\right) \frac{t_{1}-t}{\Delta t}+g\left(t_{1}\right) \frac{t-t_{0}}{\Delta t}, \quad t \in\left[t_{0}, t_{1}\right]
$$

and, on $\left[t_{j-2}, t_{j}\right], j \geq 2$,

$$
P_{2, j} g(t)=\frac{g\left(t_{j-2}\right)}{2 \Delta t^{2}}\left(t-t_{j-1}\right)\left(t-t_{j}\right)+\frac{g\left(t_{j-1}\right)}{\Delta t^{2}}\left(t-t_{j-2}\right)\left(t_{j}-t\right)+\frac{g\left(t_{j}\right)}{2 \Delta t^{2}}\left(t-t_{j-1}\right)\left(t-t_{j-2}\right) .
$$

The following error estimates hold: 


$$
g(t)-P_{1,1} g(t)=\frac{g^{\prime \prime}(\xi)}{2}\left(t-t_{0}\right)\left(t-t_{1}\right), \quad t \in\left[t_{0}, t_{1}\right], \quad \xi \in\left(t_{0}, t_{1}\right),
$$

and

$$
g(t)-P_{2, j} g(t)=\frac{g^{\prime \prime \prime}\left(\varsigma_{j}\right)}{6}\left(t-t_{j-2}\right)\left(t-t_{j-1}\right)\left(t-t_{j}\right), \quad \varsigma_{j} \in\left(t_{j-2}, t_{j}\right) .
$$

Let us now introduce the standard notations, with $j \geq 1$,

$$
t_{j-1 / 2}=\frac{t_{j}+t_{j-1}}{2}, \quad \delta_{t} g_{j-\frac{1}{2}}=\frac{g\left(t_{j}\right)-g\left(t_{j-1}\right)}{\Delta t}, \quad \delta_{t}^{2} g_{j}=\frac{\delta_{t} g_{j+\frac{1}{2}}-\delta_{t} g_{j-\frac{1}{2}}}{\Delta t} .
$$

Then we have, with $k=2,3, \ldots, N$,

$$
\begin{aligned}
{ }_{0}^{C} D_{t}^{\alpha} f\left(t_{k}\right) & =\frac{1}{\Gamma(2-\alpha)}\left(\int_{t_{0}}^{t_{1}} \frac{g^{\prime}(\tau) d \tau}{\left(t_{k}-\tau\right)^{\alpha-1}}+\sum_{j=2}^{k} \int_{t_{j-1}}^{t_{j}} \frac{g^{\prime}(\tau) d \tau}{\left(t_{k}-\tau\right)^{\alpha-1}}\right) \\
& =\frac{1}{\Gamma(2-\alpha)}\left(\int_{t_{0}}^{t_{1}} \frac{\left(P_{1,1} g(\tau)\right)^{\prime} d \tau}{\left(t_{k}-\tau\right)^{\alpha-1}}+\sum_{j=2}^{k} \int_{t_{j-1}}^{t_{j}} \frac{\left(P_{2, j} g(\tau)\right)^{\prime} d \tau}{\left(t_{k}-\tau\right)^{\alpha-1}}\right)+R_{k} \\
& =\frac{1}{\Gamma(2-\alpha)}\left(\delta_{t} g_{\frac{1}{2}} \int_{t_{0}}^{t_{1}}\left(t_{k}-\tau\right)^{1-\alpha} d \tau+\sum_{j=2}^{k} \int_{t_{j-1}}^{t_{j}}\left(t_{k}-\tau\right)^{1-\alpha}\left[\delta_{t} g_{j-\frac{1}{2}}+\left(\tau-t_{j-\frac{1}{2}}\right) \delta_{t}^{2} g_{j-1}\right] d \tau\right)+R_{k} \\
& =\frac{1}{\Gamma(2-\alpha)}\left(\sum_{j=1}^{k} \delta_{t} g_{j-\frac{1}{2}} \int_{t_{j-1}}^{t_{j}}\left(t_{k}-\tau\right)^{1-\alpha} d \tau+\sum_{j=2}^{k} \delta_{t}^{2} g_{j-1} \int_{t_{j-1}}^{t_{j}}\left(t_{k}-\tau\right)^{1-\alpha}\left(\tau-t_{j-\frac{1}{2}}\right) d \tau\right)+R_{k},
\end{aligned}
$$

where $R_{k}$ denotes the truncation error which satisfies

$$
R_{k}=\frac{1}{\Gamma(2-\alpha)}\left(\int_{t_{0}}^{t_{1}} \frac{g^{\prime}(\tau)-\left(P_{1,1} g(\tau)\right)^{\prime}}{\left(t_{k}-\tau\right)^{\alpha-1}} d \tau+\sum_{j=2}^{k} \int_{t_{j-1}}^{t_{j}} \frac{g^{\prime}(\tau)-\left(P_{2, j} g(\tau)\right)^{\prime}}{\left(t_{k}-\tau\right)^{\alpha-1}} d \tau\right) .
$$

Note that

$$
\begin{aligned}
& \int_{t_{j-1}}^{t_{j}}\left(t_{k}-\tau\right)^{1-\alpha} d \tau=\frac{1}{2-\alpha} b_{k-j}^{(\alpha)} \Delta t^{2-\alpha} \\
& \int_{t_{j-1}}^{t_{j}}\left(\tau-t_{j-\frac{1}{2}}\right)\left(t_{k}-\tau\right)^{1-\alpha} d \tau=\frac{1}{2-\alpha} e_{k-j}^{(\alpha)} \Delta t^{3-\alpha}, \quad 2 \leq j \leq k,
\end{aligned}
$$

where $b_{l}^{(\alpha)}$ and $e_{l}^{(\alpha)}, l \geq 0$ are defined by

$$
b_{l}^{(\alpha)}=(l+1)^{2-\alpha}-l^{2-\alpha},
$$

and

$$
e_{l}^{(\alpha)}=\frac{1}{3-\alpha}\left((l+1)^{3-\alpha}-l^{3-\alpha}\right)-\frac{1}{2}\left((l+1)^{2-\alpha}+l^{2-\alpha}\right) .
$$

We obtain, by (9),

$$
\begin{aligned}
{ }_{0}^{C} D_{t}^{\alpha} f\left(t_{k}\right) & =\frac{\Delta t^{2-\alpha}}{\Gamma(3-\alpha)}\left(\sum_{j=1}^{k} b_{k-j}^{(\alpha)} \delta_{t} g_{j-\frac{1}{2}}+\sum_{j=2}^{k} e_{k-j}^{(\alpha)} \delta_{t} g_{j-\frac{1}{2}}-\sum_{j=1}^{k-1} e_{k-j-1}^{(\alpha)} \delta_{t} g_{j-\frac{1}{2}}\right)+R_{k} \\
& =\frac{\Delta t^{1-\alpha}}{\Gamma(3-\alpha)}\left(w_{0}^{(\alpha)} g\left(t_{k}\right)-\sum_{j=1}^{k-1}\left(w_{k-j-1}^{(\alpha)}-w_{k-j}^{(\alpha)}\right) g\left(t_{j}\right)-w_{k-1}^{(\alpha)} g\left(t_{0}\right)\right)+R_{k},
\end{aligned}
$$


where $w_{l}^{(\alpha)}, l \geq 0$ are defined as follows:

For $k \geq 2$, we have

$$
w_{l}^{(\alpha)}= \begin{cases}b_{0}^{(\alpha)}+e_{0}^{(\alpha)}, & l=0 \\ b_{l}^{(\alpha)}+e_{l}^{(\alpha)}-e_{l-1}^{(\alpha)}, & 1 \leq l \leq k-2 \\ b_{l}^{(\alpha)}-e_{l-1}^{(\alpha)}, & l=k-1\end{cases}
$$

Hence we get, for $k \geq 2$,

$$
\begin{aligned}
& \frac{1}{2}\left({ }_{0}^{C} D_{t}^{\alpha} f\left(t_{k}\right)+{ }_{0}^{C} D_{t}^{\alpha} f\left(t_{k-1}\right)\right) \\
= & \frac{\Delta t^{1-\alpha}}{\Gamma(3-\alpha)}\left(w_{0}^{(\alpha)} \frac{g\left(t_{k}\right)+g\left(t_{k-1}\right)}{2}-\sum_{j=2}^{k-1}\left(w_{k-j-1}^{(\alpha)}-w_{k-j}^{(\alpha)}\right) \frac{g\left(t_{j}\right)+g\left(t_{j-1}\right)}{2}\right. \\
& \left.-\left(w_{k-2}^{(\alpha)}-w_{k-1}^{(\alpha)}\right) \frac{g\left(t_{1}\right)+g\left(t_{0}\right)}{2}-w_{k-1}^{(\alpha)} g\left(t_{0}\right)\right)+\frac{R_{k}+R_{k-1}}{2} .
\end{aligned}
$$

Next lemma shows the properties of the weights $w_{l}^{(\alpha)}, 0 \leq l \leq k-1$ defined in (14). For simplicity of the notations, we only consider the case $k \geq 3$ from now on. Similarly one can consider the case for $k=2$.

Lemma 2.1. Let $k \geq 3$. For any $\alpha \in(1,2)$, the weights $w_{l}^{(\alpha)}, 0 \leq l \leq k-1$ defined in (14) satisfy

$$
\begin{aligned}
& w_{0}^{(\alpha)}>\left|w_{1}^{(\alpha)}\right|, \\
& w_{l}^{(\alpha)}>0, l \neq 1, \\
& w_{2}^{(\alpha)} \geq w_{3}^{(\alpha)} \geq \cdots \geq w_{k-1}^{(\alpha)} \quad \text { for } k \geq 4, \\
& w_{0}^{(\alpha)}>w_{2}^{(\alpha)}, \\
& \sum_{l=0}^{k-1} w_{l}^{(\alpha)}=\Delta t^{2-\alpha} .
\end{aligned}
$$

Proof: The proof is similar to the proof of [10, Lemma 2.2] where the authors considered the approximation of the Caputo fractional derivative ${ }_{0}^{C} D_{t}^{\alpha} f\left(t_{k}\right)$ for $0<\alpha<1$. We omit the proof here.

To estimate $\frac{g\left(t_{j}\right)+g\left(t_{j-1}\right)}{2}, j=1,2, \ldots k, k \geq 3$ in (15), where $g(\tau)=f^{\prime}(\tau)$, we shall apply the following lemma.

Lemma 2.2. Let $k \geq 3$. Assume that $f \in C^{4}[0, T]$, then we have

$$
\begin{aligned}
& \frac{f_{j}^{\prime}+f_{j-1}^{\prime}}{2}=\frac{1}{12}\left(13 \delta_{t} f_{j-\frac{1}{2}}-2 \delta_{t} f_{j+\frac{1}{2}}+\delta_{t} f_{j+\frac{3}{2}}\right)+O\left(\Delta t^{3}\right), \quad j=1, \\
& \frac{f_{j}^{\prime}+f_{j-1}^{\prime}}{2}=\frac{1}{12}\left(\delta_{t} f_{j+\frac{1}{2}}+10 \delta_{t} f_{j-\frac{1}{2}}+\delta_{t} f_{j-\frac{3}{2}}\right)+O\left(\Delta t^{4}\right), \quad 2 \leq j \leq k-1, \\
& \frac{f_{j}^{\prime}+f_{j-1}^{\prime}}{2}=\frac{1}{12}\left(13 \delta_{t} f_{j-\frac{1}{2}}-2 \delta_{t} f_{j-\frac{3}{2}}+\delta_{t} f_{j-\frac{5}{2}}\right)+O\left(\Delta t^{3}\right) . \quad j=k .
\end{aligned}
$$

Proof: See the Appendix. 
Applying Lemma 2.2, we have, by (15), with $k \geq 3$,

$$
\begin{aligned}
& \frac{1}{2}\left({ }_{0}^{C} D_{t}^{\alpha} f\left(t_{k}\right)+{ }_{0}^{C} D_{t}^{\alpha} f\left(t_{k-1}\right)\right) \\
= & \frac{\Delta t^{1-\alpha}}{\Gamma(3-\alpha)}\left[\frac{1}{12} w_{0}^{(\alpha)}\left(13 \delta_{t} f_{k-\frac{1}{2}}-2 \delta_{t} f_{k-\frac{3}{2}}+\delta_{t} f_{k-\frac{5}{2}}\right)\right. \\
& -\sum_{j=2}^{k-1} \frac{1}{12}\left(w_{k-j-1}^{(\alpha)}-w_{k-j}^{(\alpha)}\right)\left(\delta_{t} f_{j+\frac{1}{2}}+10 \delta_{t} f_{j-\frac{1}{2}}+\delta_{t} f_{j-\frac{3}{2}}\right) \\
& \left.-\frac{1}{12}\left(w_{k-2}^{(\alpha)}-w_{k-1}^{(\alpha)}\right)\left(13 \delta_{t} f_{\frac{1}{2}}-2 \delta_{t} f_{\frac{3}{2}}+\delta_{t} f_{\frac{5}{2}}\right)-w_{k-1}^{(\alpha)} f^{\prime}\left(t_{0}\right)\right] \\
& +\frac{\Delta t^{1-\alpha}}{\Gamma(3-\alpha)}\left[w_{0}^{(\alpha)} O\left(\Delta t^{3}\right)-\sum_{j=2}^{k-1}\left(w_{k-j-1}^{(\alpha)}-w_{k-j}^{(\alpha)}\right) O\left(\Delta t^{4}\right)-\left(w_{k-2}^{(\alpha)}-w_{k-1}^{(\alpha)}\right) O\left(\Delta t^{3}\right)\right]+\frac{R_{k}+R_{k-1}}{2} \\
= & \frac{\Delta t^{-\alpha}}{\Gamma(3-\alpha)}\left(\sum_{j=0}^{k} d_{j} f_{k-j}-w_{k-1}^{(\alpha)} f^{\prime}\left(t_{0}\right)\right)+R^{k-\frac{1}{2}},
\end{aligned}
$$

where, by using the properties of the weights in Lemma 2.1,

$$
\begin{aligned}
R^{k-\frac{1}{2}} & =\frac{\Delta t^{1-\alpha}}{\Gamma(3-\alpha)}\left(w_{0}^{(\alpha)} O\left(\Delta t^{3}\right)-\sum_{j=2}^{k-1}\left(w_{k-j-1}^{(\alpha)}-w_{k-j}^{(\alpha)}\right) O\left(\Delta t^{4}\right)-\left(w_{k-2}^{(\alpha)}-w_{k-1}^{(\alpha)}\right) O\left(\Delta t^{3}\right)\right)+\frac{R_{k}+R_{k-1}}{2} \\
& =O\left(\Delta t^{4-\alpha}\right)+\frac{R_{k}+R_{k-1}}{2} .
\end{aligned}
$$

Here $d_{k}$ are determined by the following formula:

For $k=3$,

$$
\left\{\begin{aligned}
d_{0} & =w_{0}^{(\alpha)}+\frac{1}{12} w_{2}^{(\alpha)}, \\
d_{1} & =-2 w_{0}^{(\alpha)}+w_{1}^{(\alpha)}-\frac{1}{4} w_{2}^{(\alpha)}, \\
d_{2} & =w_{0}^{(\alpha)}-2 w_{1}^{(\alpha)}+\frac{5}{4} w_{2}^{(\alpha)}, \\
d_{3} & =w_{1}^{(\alpha)}-\frac{13}{12} w_{2}^{(\alpha)} .
\end{aligned}\right.
$$

For $k=4$,

For $k=5$,

$$
\left\{\begin{aligned}
d_{0} & =w_{0}^{(\alpha)}+\frac{1}{12} w_{1}^{(\alpha)} \\
d_{1} & =-2 w_{0}^{(\alpha)}+\frac{2}{3} w_{1}^{(\alpha)}+\frac{1}{12} w_{3}^{(\alpha)} \\
d_{2} & =w_{0}^{(\alpha)}-\frac{3}{2} w_{1}^{(\alpha)}+w_{2}^{(\alpha)}-\frac{1}{4} w_{3}^{(\alpha)} \\
d_{3} & =\frac{2}{3} w_{1}^{(\alpha)}-2 w_{2}^{(\alpha)}+\frac{5}{4} w_{3}^{(\alpha)} \\
d_{4} & =\frac{1}{12} w_{1}^{(\alpha)}+w_{2}^{(\alpha)}-\frac{13}{12} w_{3}^{(\alpha)} .
\end{aligned}\right.
$$

$$
\left\{\begin{aligned}
d_{0} & =w_{0}^{(\alpha)}+\frac{1}{12} w_{1}^{(\alpha)} \\
d_{1} & =-2 w_{0}^{(\alpha)}+\frac{2}{3} w_{1}^{(\alpha)}+\frac{1}{12} w_{2}^{(\alpha)} \\
d_{2} & =w_{0}^{(\alpha)}-\frac{3}{2} w_{1}^{(\alpha)}+\frac{2}{3} w_{2}^{(\alpha)}+\frac{1}{12} w_{4}^{(\alpha)} \\
d_{3} & =\frac{2}{3} w_{1}^{(\alpha)}-\frac{3}{2} w_{2}^{(\alpha)}+w_{3}^{(\alpha)}-\frac{1}{4} w_{4}^{(\alpha)} \\
d_{4} & =\frac{1}{12} w_{1}^{(\alpha)}+\frac{2}{3} w_{2}^{(\alpha)}-2 w_{3}^{(\alpha)}+\frac{5}{4} w_{4}^{(\alpha)} \\
d_{5} & =\frac{1}{12} w_{2}^{(\alpha)}+w_{3}^{(\alpha)}-\frac{13}{12} w_{4}^{(\alpha)} .
\end{aligned}\right.
$$


For $k=6$,

$$
\left\{\begin{aligned}
d_{0} & =w_{0}^{(\alpha)}+\frac{1}{12} w_{1}^{(\alpha)} \\
d_{1} & =-2 w_{0}^{(\alpha)}+\frac{2}{3} w_{1}^{(\alpha)}+\frac{1}{12} w_{2}^{(\alpha)} \\
d_{2} & =w_{0}^{(\alpha)}-\frac{3}{2} w_{1}^{(\alpha)}+\frac{2}{3} w_{2}^{(\alpha)}+\frac{1}{12} w_{3}^{(\alpha)} \\
d_{3} & =\frac{2}{3} w_{1}^{(\alpha)}-\frac{3}{2} w_{2}^{(\alpha)}+\frac{2}{3} w_{3}^{(\alpha)}+\frac{1}{12} w_{5}^{(\alpha)} \\
d_{4} & =\frac{1}{12} w_{1}^{(\alpha)}+\frac{2}{3} w_{2}^{(\alpha)}-\frac{3}{2} w_{3}^{(\alpha)}+w_{4}^{(\alpha)}-\frac{1}{4} w_{5}^{(\alpha)} \\
d_{5} & =\frac{1}{12} w_{2}^{(\alpha)}+\frac{2}{3} w_{3}^{(\alpha)}-2 w_{4}^{(\alpha)}+\frac{5}{4} w_{5}^{(\alpha)} \\
d_{6} & =\frac{1}{12} w_{3}^{(\alpha)}+w_{4}^{(\alpha)}-\frac{13}{12} w_{5}^{(\alpha)}
\end{aligned}\right.
$$

For $k \geq 7$,

$$
\left\{\begin{array}{l}
d_{0}=w_{0}^{(\alpha)}+\frac{1}{12} w_{1}^{(\alpha)} \\
d_{1}=-2 w_{0}^{(\alpha)}+\frac{2}{3} w_{1}^{(\alpha)}+\frac{1}{12} w_{2}^{(\alpha)} \\
d_{2}=w_{0}^{(\alpha)}-\frac{3}{2} w_{1}^{(\alpha)}+\frac{2}{3} w_{2}^{(\alpha)}+\frac{1}{12} w_{3}^{(\alpha)} \\
d_{3}=\frac{2}{3} w_{1}^{(\alpha)}-\frac{3}{2} w_{2}^{(\alpha)}+\frac{2}{3} w_{3}^{(\alpha)}+\frac{1}{12} w_{4}^{(\alpha)} \\
d_{j}=\frac{1}{12} w_{j-3}^{(\alpha)}+\frac{2}{3} w_{j-2}^{(\alpha)}-\frac{3}{2} w_{j-1}^{(\alpha)}+\frac{2}{3} w_{j}^{(\alpha)}+\frac{1}{12} w_{j+1}^{(\alpha)}(4 \leq j \leq k-4), \\
d_{k-3}=\frac{1}{12} w_{k-6}^{(\alpha)}+\frac{2}{3} w_{k-5}^{(\alpha)}-\frac{3}{2} w_{k-4}^{(\alpha)}+\frac{2}{3} w_{k-3}^{(\alpha)}+\frac{1}{12} w_{k-1}^{(\alpha)}, \\
d_{k-2}=\frac{1}{12} w_{k-5}^{(\alpha)}+\frac{2}{3} w_{k-4}^{(\alpha)}-\frac{3}{2} w_{k-3}^{(\alpha)}+w_{k-2}^{(\alpha)}-\frac{1}{4} w_{k-1}^{(\alpha)}, \\
d_{k-1}=\frac{1}{12} w_{k-4}^{(\alpha)}+\frac{2}{3} w_{k-3}^{(\alpha)}-2 w_{k-2}^{(\alpha)}+\frac{5}{4} w_{k-1}^{(\alpha)}, \\
d_{k}=\frac{1}{12} w_{k-3}^{(\alpha)}+w_{k-2}^{(\alpha)}-\frac{13}{12} w_{k-1}^{(\alpha)} .
\end{array}\right.
$$

Now we turn to the truncation error $R^{k-\frac{1}{2}}$ in $(25)$. We have

Theorem 2.3. Let $k \geq 3$. Assume that $f \in C^{4}[0, T]$. For any $\alpha \in(1,2)$, the truncation error $R^{k-\frac{1}{2}}$ defined in (25) satisfies

$$
\begin{aligned}
\left|R^{k-\frac{1}{2}}\right| \leq & C \Delta t^{4-\alpha}+\frac{1}{\Gamma(2-\alpha)}\left(\frac{\alpha-1}{12} \max _{t_{0} \leq t \leq t_{1}}\left|f^{(3)}(t)\right| \Delta t^{3-\alpha}\right. \\
& \left.+\left(\frac{1}{12}+\frac{(\alpha-1)(6-\alpha)}{6(2-\alpha)(3-\alpha)(4-\alpha)}\right) \max _{t_{0} \leq t \leq t_{k}}\left|f^{(4)}(t)\right| \Delta t^{4-\alpha}\right) .
\end{aligned}
$$

Proof: By (7) and (8), we have

$$
\begin{aligned}
\left|R_{k}\right| & =\left|\frac{1}{\Gamma(2-\alpha)}\left(\int_{t_{0}}^{t_{1}}\left(g(\tau)-P_{1,1} g(\tau)\right)^{\prime}\left(t_{k}-\tau\right)^{1-\alpha} d \tau+\sum_{j=2}^{k} \int_{t_{j-1}}^{t_{j}}\left(g(\tau)-P_{2, j} g(\tau)\right)^{\prime}\left(t_{k}-\tau\right)^{1-\alpha} d \tau\right)\right| \\
& =\left|\frac{\alpha-1}{\Gamma(2-\alpha)}\left(\int_{t_{0}}^{t_{1}}\left(g(\tau)-P_{1,1} g(\tau)\right)\left(t_{k}-\tau\right)^{-\alpha} d \tau+\sum_{j=2}^{k} \int_{t_{j-1}}^{t_{j}}\left(g(\tau)-P_{2, j} g(\tau)\right)\left(t_{k}-\tau\right)^{-\alpha} d \tau\right)\right|,
\end{aligned}
$$

where

$$
\begin{aligned}
\left|\int_{t_{0}}^{t_{1}}\left(g(\tau)-P_{1,1} g(\tau)\right)\left(t_{k}-\tau\right)^{-\alpha} d \tau\right| & =\frac{1}{2}\left|\int_{t_{0}}^{t_{1}} g^{\prime \prime}(\xi)\left(\tau-t_{0}\right)\left(\tau-t_{1}\right)\left(t_{k}-\tau\right)^{-\alpha} d \tau\right| \\
& \leq \frac{1}{12} \max _{t_{0} \leq t \leq t_{1}}\left|f^{(3)}(t)\right|\left(t_{k}-t_{1}\right)^{-\alpha} \Delta t^{3} \\
& \leq \frac{1}{12} \max _{t_{0} \leq t \leq t_{1}}\left|f^{(3)}(t)\right| \Delta t^{3-\alpha}, \quad \xi \in\left(t_{0}, t_{1}\right)
\end{aligned}
$$


and

$$
\begin{aligned}
\left|\sum_{j=2}^{k-1} \int_{t_{j-1}}^{t_{j}}\left(g(\tau)-P_{2, j} g(\tau)\right)\left(t_{k}-\tau\right)^{-\alpha} d \tau\right| & =\frac{1}{6}\left|\sum_{j=2}^{k-1} \int_{t_{j-1}}^{t_{j}} g^{(3)}\left(\varsigma_{j}\right)\left(\tau-t_{j-2}\right)\left(\tau-t_{j-1}\right)\left(\tau-t_{j}\right)\left(t_{k}-\tau\right)^{-\alpha} d \tau\right| \\
& \leq \frac{1}{12(\alpha-1)} \max _{t_{0} \leq t \leq t_{k-1}}\left|f^{(4)}(t)\right| \Delta t^{4-\alpha}, \quad \varsigma_{j} \in\left(t_{j-2}, t_{j}\right)
\end{aligned}
$$

and

$$
\begin{aligned}
\int_{t_{k-1}}^{t_{k}}\left(g(\tau)-P_{2, k} g(\tau)\right)\left(t_{k}-\tau\right)^{-\alpha} d \tau & =-\frac{1}{6} g^{(3)}\left(\varsigma_{k}\right) \int_{t_{k-1}}^{t_{k}}\left(\tau-t_{k-2}\right)\left(\tau-t_{k-1}\right)\left(t_{k}-\tau\right)^{1-\alpha} d \tau \\
& =-\frac{6-\alpha}{6(2-\alpha)(3-\alpha)(4-\alpha)} f^{(4)}\left(\varsigma_{k}\right) \Delta t^{4-\alpha}, \quad \varsigma_{k} \in\left(t_{k-2}, t_{k}\right) .
\end{aligned}
$$

Substituting (29), (30) and (31) into (28), we get

$$
\left|R_{k}\right| \leq \frac{1}{\Gamma(2-\alpha)}\left[\frac{\alpha-1}{12} \max _{t_{0} \leq t \leq t_{1}}\left|f^{(3)}(t)\right|\left(t_{k}-t_{1}\right)^{-\alpha} \Delta t^{3}+\left(\frac{1}{12}+\frac{(6-\alpha)(\alpha-1)}{6(2-\alpha)(3-\alpha)(4-\alpha)}\right) \max _{t_{0} \leq t \leq t_{k}}\left|f^{(4)}(t)\right| \Delta t^{4-\alpha}\right],
$$

which implies that

$$
\begin{aligned}
\left|\frac{R_{k}+R_{k-1}}{2}\right| \leq & \frac{1}{\Gamma(2-\alpha)}\left[\frac{\alpha-1}{12} \max _{t_{0} \leq t \leq t_{1}}\left|f^{(3)}(t)\right| \frac{\left(t_{k}-t_{1}\right)^{-\alpha}+\left(t_{k-1}-t_{1}\right)^{-\alpha}}{2} \Delta t^{3}\right. \\
& \left.+\left(\frac{1}{12}+\frac{(6-\alpha)(\alpha-1)}{6(2-\alpha)(3-\alpha)(4-\alpha)}\right) \max _{t_{0} \leq t \leq t_{k}}\left|f^{(4)}(t)\right| \Delta t^{4-\alpha}\right] \\
\leq & \frac{1}{\Gamma(2-\alpha)}\left[\frac{\alpha-1}{12} \max _{t_{0} \leq t \leq t_{1}}\left|f^{(3)}(t)\right|\left(t_{k-1}-t_{1}\right)^{-\alpha} \Delta t^{3}\right. \\
& \left.+\left(\frac{1}{12}+\frac{(6-\alpha)(\alpha-1)}{6(2-\alpha)(3-\alpha)(4-\alpha)}\right) \max _{t_{0} \leq t \leq t_{k}}\left|f^{(4)}(t)\right| \Delta t^{4-\alpha}\right] .
\end{aligned}
$$

Together these estimates with (25) completes the proof of Theorem 2.3.

Next we will show that when $f^{\prime \prime \prime}\left(t_{0}\right)=0$, the approximate scheme $(24)$ actually has the convergence order $O\left(\Delta t^{4-\alpha}\right), 1<\alpha<2$ with $k \geq 3$. To see this, let us assume that $g(t)$ is well defined on $t \in\left[t_{-1}, T\right]$ and we approximate $g$ in (6) by using the quadratic interpolation polynomial $P_{2, j} g$ on all the subinterval $\left[t_{j-1}, t_{j}\right], j \geq 1$. (Recall that, in $(9)$, we approximate $g$ on $\left[t_{0}, t_{1}\right]$ by using the linear interpolation polynomial $P_{1,1} g$ and by $P_{2, j} g$ on other subintervals.) Then we have, with $k=2,3, \ldots, N$,

$$
\begin{aligned}
{ }_{0}^{C} D_{t}^{\alpha} f\left(t_{k}\right) & =\frac{1}{\Gamma(2-\alpha)} \sum_{j=1}^{k} \int_{t_{j-1}}^{t_{j}} \frac{g^{\prime}(\tau) d \tau}{\left(t_{k}-\tau\right)^{\alpha-1}} \\
& =\frac{1}{\Gamma(2-\alpha)} \sum_{j=1}^{k} \int_{t_{j-1}}^{t_{j}} \frac{\left(P_{2, j} g(\tau)\right)^{\prime} d \tau}{\left(t_{k}-\tau\right)^{\alpha-1}}+\bar{R}_{k} \\
& =\frac{1}{\Gamma(2-\alpha)}\left(\sum_{j=1}^{k} \int_{t_{j-1}}^{t_{j}}\left(t_{k}-\tau\right)^{1-\alpha}\left[\delta_{t} g_{j-\frac{1}{2}}+\left(\tau-t_{j-\frac{1}{2}}\right) \delta_{t}^{2} g_{j-1}\right] d \tau\right)+\bar{R}_{k} \\
& =\frac{1}{\Gamma(2-\alpha)}\left(\sum_{j=1}^{k} \delta_{t} g_{j-\frac{1}{2}} \int_{t_{j-1}}^{t_{j}}\left(t_{k}-\tau\right)^{1-\alpha} d \tau+\sum_{j=1}^{k} \delta_{t}^{2} g_{j-1} \int_{t_{j-1}}^{t_{j}}\left(t_{k}-\tau\right)^{1-\alpha}\left(\tau-t_{j-\frac{1}{2}}\right) d \tau\right)+\bar{R}_{k},
\end{aligned}
$$


where

$$
\bar{R}_{k}=\frac{1}{\Gamma(2-\alpha)} \sum_{j=1}^{k} \int_{t_{j-1}}^{t_{j}} \frac{g^{\prime}(\tau)-\left(P_{2, j} g(\tau)\right)^{\prime}}{\left(t_{k}-\tau\right)^{\alpha-1}} d \tau .
$$

After some calculations, we obtain, with $k \geq 2$,

$$
\begin{aligned}
{ }_{0}^{C} D_{t}^{\alpha} f\left(t_{k}\right) & =\frac{\Delta t^{2-\alpha}}{\Gamma(3-\alpha)}\left(\sum_{j=1}^{k} b_{k-j}^{(\alpha)} \delta_{t} g_{j-\frac{1}{2}}+\sum_{j=1}^{k} e_{k-j}^{(\alpha)} \delta_{t} g_{j-\frac{1}{2}}-\sum_{j=0}^{k-1} e_{k-j-1}^{(\alpha)} \delta_{t} g_{j-\frac{1}{2}}\right)+\bar{R}_{k} \\
& =\frac{\Delta t^{1-\alpha}}{\Gamma(3-\alpha)}\left(w_{0}^{(\alpha)} g\left(t_{k}\right)-\sum_{j=1}^{k-1}\left(w_{k-j-1}^{(\alpha)}-w_{k-j}^{(\alpha)}\right) g\left(t_{j}\right)-w_{k-1}^{(\alpha)} g\left(t_{0}\right)-e_{k-1}^{(\alpha)}\left(g\left(t_{1}\right)+g\left(t_{-1}\right)-2 g_{0}\right)\right)+\bar{R}_{k} \\
& =\frac{\Delta t^{1-\alpha}}{\Gamma(3-\alpha)}\left(w_{0}^{(\alpha)} g\left(t_{k}\right)-\sum_{j=1}^{k-1}\left(w_{k-j-1}^{(\alpha)}-w_{k-j}^{(\alpha)}\right) g\left(t_{j}\right)-w_{k-1}^{(\alpha)} g\left(t_{0}\right)\right)+R_{k}
\end{aligned}
$$

where $b_{l}^{(\alpha)}, e_{l}^{(\alpha)}, w_{l}^{(\alpha)}$ are defined as in (13) and

$$
R_{k}=-\frac{\Delta t^{1-\alpha}}{\Gamma(3-\alpha)} e_{k-1}^{(\alpha)}\left(g\left(t_{1}\right)+g\left(t_{-1}\right)-2 g\left(t_{0}\right)\right)+\bar{R}_{k} .
$$

We remark that for simplicity, we use the same notation $R_{k}$ in both (10) and (37) although it takes the different formulas in these two different cases.

We therefore obtain the same approximation formula as in (24) and (25), but with the different $R_{k}$. We have the following theorem:

Theorem 2.4. Let $k \geq 3$. Assume that $f \in C^{4}\left[t_{-1}, T\right]$ and $f^{\prime \prime \prime}\left(t_{0}\right)=0$. Let $R^{k-\frac{1}{2}}$ be the truncation error defined in (25) where $R_{k}$ is defined by (37). Then we have, with $\alpha \in(1,2)$,

$$
\left|R^{k-\frac{1}{2}}\right| \leq C \Delta t^{4-\alpha}+\frac{1}{\Gamma(2-\alpha)}\left(\frac{1}{12}+\frac{(\alpha-1)(6-\alpha)}{6(2-\alpha)(3-\alpha)(4-\alpha)}\right) \max _{t_{0} \leq t \leq t_{k}}\left|f^{(4)}(t)\right| \Delta t^{4-\alpha} .
$$

Proof: By (37), we have

$$
R_{k}=-\frac{\Delta t^{1-\alpha}}{\Gamma(3-\alpha)} e_{k-1}^{(\alpha)}\left(g\left(t_{1}\right)+g\left(t_{-1}\right)-2 g\left(t_{0}\right)\right)+\bar{R}_{k},
$$

where $\bar{R}_{k}$ is defined by (35). Since $g^{\prime \prime}\left(t_{0}\right)=f^{\prime \prime \prime}\left(t_{0}\right)=0$, we have, by Taylor expansion at $t_{0}$, with $\xi \in\left(t_{-1}, t_{1}\right)$,

$$
g\left(t_{1}\right)+g\left(t_{-1}\right)-2 g\left(t_{0}\right)=g^{\prime \prime}\left(t_{0}\right) \Delta t^{2}+\frac{1}{6} g^{(4)}(\xi) \Delta t^{4}=\frac{1}{6} g^{(4)}(\xi) \Delta t^{4} .
$$

Further we have, by (12), with some suitable positive constant $C$,

$$
\begin{aligned}
\left|e_{k-1}^{(\alpha)}\right| & =\left|\frac{1}{3-\alpha}\left(k^{3-\alpha}-(k-1)^{3-\alpha}\right)-\frac{1}{2}\left(k^{2-\alpha}+(k-1)^{2-\alpha}\right)\right| \\
& =\left|\frac{1}{3-\alpha} \int_{k}^{k+1}(3-\alpha) s^{2-\alpha} d s-\frac{1}{2}\left(k^{2-\alpha}+(k-1)^{2-\alpha}\right)\right| \\
& \leq C(k+1)^{2-\alpha} .
\end{aligned}
$$


Thus, noting that $k \Delta t \leq T$ for $k=3,4, \ldots, N$,

$$
\begin{aligned}
\left|R_{k}\right| & \leq\left|\frac{\Delta t^{1-\alpha}}{\Gamma(3-\alpha)} e_{k-1}^{(\alpha)}\left(g\left(t_{1}\right)+g\left(t_{-1}\right)-2 g\left(t_{0}\right)\right)\right|+\left|\bar{R}_{k}\right| \\
& \leq C(k+1)^{2-\alpha} \Delta t^{1-\alpha} \Delta t^{4}+\left|\bar{R}_{k}\right| \leq C \Delta t^{3}+\left|\bar{R}_{k}\right| \\
& \leq C \Delta t^{3}+\left|\frac{1}{\Gamma(2-\alpha)} \sum_{j=1}^{k} \int_{t_{j-1}}^{t_{j}}\left(g(\tau)-P_{2, j} g(\tau)\right)^{\prime}\left(t_{k}-\tau\right)^{1-\alpha} d \tau\right| \\
& =C \Delta t^{3}+\left|\frac{\alpha-1}{\Gamma(2-\alpha)} \sum_{j=1}^{k} \int_{t_{j-1}}^{t_{j}}\left(g(\tau)-P_{2, j} g(\tau)\right)\left(t_{k}-\tau\right)^{-\alpha} d \tau\right|,
\end{aligned}
$$

Note that

$$
\begin{aligned}
\left|\sum_{j=1}^{k-1} \int_{t_{j-1}}^{t_{j}}\left(g(\tau)-P_{2, j} g(\tau)\right)\left(t_{k}-\tau\right)^{-\alpha} d \tau\right| & =\frac{1}{6}\left|\sum_{j=1}^{k-1} \int_{t_{j-1}}^{t_{j}} g^{(3)}\left(\varsigma_{j}\right)\left(\tau-t_{j-2}\right)\left(\tau-t_{j-1}\right)\left(\tau-t_{j}\right)\left(t_{k}-\tau\right)^{-\alpha} d \tau\right| \\
& \leq \frac{1}{12(\alpha-1)} \max _{t_{-1} \leq t \leq t_{k-1}}\left|f^{(4)}(t)\right| \Delta t^{4-\alpha}, \quad \varsigma_{j} \in\left(t_{j-2}, t_{j}\right)
\end{aligned}
$$

and

$$
\begin{aligned}
\int_{t_{k-1}}^{t_{k}}\left(g(\tau)-P_{2, k} g(\tau)\right)\left(t_{k}-\tau\right)^{-\alpha} d \tau & =-\frac{1}{6} g^{(3)}\left(\varsigma_{k}\right) \int_{t_{k-1}}^{t_{k}}\left(\tau-t_{k-2}\right)\left(\tau-t_{k-1}\right)\left(t_{k}-\tau\right)^{1-\alpha} d \tau \\
& =-\frac{6-\alpha}{6(2-\alpha)(3-\alpha)(4-\alpha)} f^{(4)}\left(\varsigma_{k}\right) \Delta t^{4-\alpha}, \quad \varsigma_{k} \in\left(t_{k-2}, t_{k}\right) .
\end{aligned}
$$

Substituting (40) and (41) into (39), we get

$$
\left|R_{k}\right| \leq C \Delta t^{3}+\frac{1}{\Gamma(2-\alpha)}\left(\frac{1}{12}+\frac{(6-\alpha)(\alpha-1)}{6(2-\alpha)(3-\alpha)(4-\alpha)}\right) \max _{t_{-1} \leq t \leq t_{k}}\left|f^{(4)}(t)\right| \Delta t^{4-\alpha},
$$

which implies that

$$
\left|\frac{R_{k}+R_{k-1}}{2}\right| \leq C \Delta t^{3}+\frac{1}{\Gamma(2-\alpha)}\left(\frac{1}{12}+\frac{(6-\alpha)(\alpha-1)}{6(2-\alpha)(3-\alpha)(4-\alpha)}\right) \max _{t_{-1} \leq t \leq t_{k}}\left|f^{(4)}(t)\right| \Delta t^{4-\alpha}
$$

Together these estimates with (25) completes the proof of Theorem 2.4.

Remark 2.1. By Theorem 2.4, when $f^{\prime \prime \prime}\left(t_{0}\right)=0$ we obtain a high-order scheme $O\left(\Delta t^{4-\alpha}\right), 1<\alpha<2$ to approximate $\frac{1}{2}\left({ }_{0}^{C} D_{t}^{\alpha} f\left(t_{k}\right)+{ }_{0}^{C} D_{t}^{\alpha} f\left(t_{k-1}\right)\right)$ with $k \geq 3$. For $k=1,2$, we may approximate ${ }_{0}^{C} D_{t}^{\alpha} f\left(t_{k}\right)$ separately. In general, we may approximate ${ }_{0}^{C} D_{t}^{\alpha} f\left(t_{k}\right), k=1,2$ by using some lower order approximate schemes with the partition $0=\tilde{t}_{0}<\tilde{t}_{1}<\cdots<\tilde{t}_{M}=t_{k}, k=1,2$ and the step size $\widetilde{\Delta t}$ for some suitable positive integer $M$. We may choose $\widetilde{\Delta t}=\Delta t^{\gamma}$ for some suitable $\gamma>1$ such that the required convergence order $O\left(\Delta t^{4-\alpha}\right)$ of the approximation of the Caputo fractional derivative ${ }_{0}^{C} D_{t}^{\alpha} f\left(t_{k}\right)$ with $k=1,2$ is reached. In some special cases, we may use some simple ways to approximate the Caputo fractional derivative ${ }_{0}^{C} D_{t}^{\alpha} f\left(t_{k}\right)$ with $k=1,2$. For example, when $f^{\prime \prime \prime}\left(t_{0}\right)=0$, we may use the following way to approximate ${ }_{0}^{C} D_{t}^{\alpha} f(t)$ at $t=t_{1}$.

$$
{ }_{0}^{C} D_{t}^{\alpha} f\left(t_{1}\right)=\frac{1}{\Gamma(2-\alpha)} \int_{t_{0}}^{t_{1}}\left(t_{1}-s\right)^{1-\alpha} f^{\prime \prime}(s) d s \approx \frac{1}{\Gamma(2-\alpha)} \int_{t_{0}}^{t_{1}}\left(t_{1}-s\right)^{1-\alpha} f^{\prime \prime}\left(t_{0}\right) d s=\frac{f^{\prime \prime}\left(t_{0}\right)}{\Gamma(3-\alpha)} t_{1}^{2-\alpha},
$$


which has the convergence order

$$
\begin{aligned}
& { }_{0}^{C} D_{t}^{\alpha} f\left(t_{1}\right)-\frac{f^{\prime \prime}\left(t_{0}\right)}{\Gamma(3-\alpha)} t_{1}^{2-\alpha}=\frac{1}{\Gamma(2-\alpha)} \int_{t_{0}}^{t_{1}}\left(t_{1}-s\right)^{1-\alpha}\left(f^{\prime \prime}(s)-f^{\prime \prime}\left(t_{0}\right)\right) d s \\
& =\frac{1}{\Gamma(2-\alpha)} \int_{t_{0}}^{t_{1}}\left(t_{1}-s\right)^{1-\alpha} f^{(4)}(\xi) s^{2} d s=O\left(\Delta t^{4-\alpha}\right), \xi \in\left(t_{0}, t_{1}\right) .
\end{aligned}
$$

Similarly we may approximate ${ }_{0}^{C} D_{t}^{\alpha} f(t)$ at $t=t_{2}$ by using the same idea.

\section{A high-order numerical method for solving fractional diffusion wave equation}

In this section, we will introduce a new finite difference method for solving fractional diffusion wave equation (2)-(4) with the convergence order $O\left(\Delta t^{4-\alpha}+h^{2}\right), 1<\alpha<2$, where $\Delta t$ and $h$ denote the time and space step sizes, respectively.

Let $0=x_{0}<x_{1}<\cdots<x_{M}=1$ be the space partition on $[0,1]$ and $h$ the space step size. At $\left(x_{i}, t_{k}\right)$ with $i=1,2, \ldots, M-1$ and $k \geq 3$, we use the central difference scheme to approximate the second order spatial derivative

$$
\frac{\partial^{2} u\left(x_{i}, t_{k}\right)}{\partial x^{2}}=\frac{u\left(x_{i+1}, t_{k}\right)-2 u\left(x_{i}, t_{k}\right)+u\left(x_{i-1}, t_{k}\right)}{h^{2}}+O\left(h^{2}\right),
$$

and use (24) to approximate the time Caputo fractional derivative, that is, with $k \geq 3$,

$$
\begin{aligned}
& \frac{1}{2}\left({ }_{0}^{C} D_{t}^{\alpha} u\left(x_{i}, t_{k}\right)+{ }_{0}^{C} D_{t}^{\alpha} u\left(x_{i}, t_{k-1}\right)\right) \\
= & \frac{\Delta t^{-\alpha}}{\Gamma(3-\alpha)}\left(d_{0} u\left(x_{i}, t_{k}\right)+d_{1} u\left(x_{i}, t_{k-1}\right)+d_{2} u\left(x_{i}, t_{k-2}\right)+d_{3} u\left(x_{i}, t_{k-3}\right)+\sum_{j=4}^{k-4} d_{j} u\left(x_{i}, t_{k-j}\right)\right. \\
& \left.+d_{k-3} u\left(x_{i}, t_{3}\right)+d_{k-2} u\left(x_{i}, t_{2}\right)+d_{k-1} u\left(x_{i}, t_{1}\right)+d_{k} u\left(x_{i}, t_{0}\right)-w_{k-1}^{(\alpha)} u_{1}\left(x_{i}\right)\right)+O\left(\Delta t^{4-\alpha}\right) .
\end{aligned}
$$

Omitting the truncation error terms in (45) and (46), and replacing $u\left(x_{i}, t_{k}\right)$ by its numerical approximation $U_{i}^{k}$, we define the following finite difference scheme for solving (2)-(4), with $k \geq 3,1<i<M-1$,

$$
\begin{aligned}
& \frac{\Delta t^{-\alpha}}{\Gamma(3-\alpha)}\left(\sum_{j=0}^{k} d_{j} U_{i}^{k-j}-w_{k-1}^{(\alpha)} u_{1}\left(x_{i}\right)\right) \\
& \quad=\frac{1}{2}\left(\frac{U_{i+1}^{k}-2 U_{i}^{k}+U_{i-1}^{k}}{h^{2}}+\frac{U_{i+1}^{k-1}-2 U_{i}^{k-1}+U_{i-1}^{k-1}}{h^{2}}\right)+\frac{F_{i}^{k}+F_{i}^{k-1}}{2}, \\
& U_{i}^{0}=u_{0}\left(x_{i}\right), \quad 0 \leq i \leq M-1, \\
& U_{0}^{k}=\phi\left(t_{k}\right), \quad U_{M}^{k}=\varphi\left(t_{k}\right) .
\end{aligned}
$$

Remark 3.1. Note that the numerical method (47)-(49) are defined for $k \geq 3$ which means that we need to determine the starting approximations $U_{i}^{k}$ with $k=1,2$ and $i=0,1, \cdots, M$ before we use this method to calculate the approximations $U_{i}^{k}$ for $k \geq 3$. We may use other numerical methods to obtain the starting approximations $U_{i}^{k}$ with $k=1,2$ and $i=0,1, \ldots, M$ with the required accuracy.

The following two lemmas provide the properties of the weights $d_{j}$ in the numerical method (47)-(49).

Lemma 3.1. Let $k=3$. We have

$$
d_{0}>0, \quad d_{1}<0, \quad d_{2}>0, \quad d_{3}<0, \quad d_{1}+d_{2}+d_{3}=-d_{0} .
$$

Proof: The proof is obvious and we omit the proof here. 
Lemma 3.2. Let $k \geq 4$. The weights $d_{j}, 0 \leq j \leq k$ in (47)-(49) satisfy the following properties

$$
\begin{aligned}
& d_{0}=\frac{5}{6}+\frac{1}{24} \cdot 2^{2-\alpha}+\frac{1}{12(3-\alpha)} \cdot 2^{3-\alpha} \in(1.0833,1.4583), \\
& d_{1}, d_{3}<0, \\
& d_{j}>0, \quad j \neq 1,3,4, \\
& d_{4}<0 \text { for } k=5, \quad \text { while } d_{4}>0 \text { for } k \neq 5 \text { and } \alpha \in(1,1.5878), \\
& \sum_{j=0}^{k} d_{j}=0 .
\end{aligned}
$$

Proof: The estimate (50) is obvious and we omit this proof here. Let us now consider (51). For $k=4$, we have

$$
\begin{aligned}
d_{1} & =-\left(\frac{5}{3}-\frac{3}{8} \cdot 2^{2-\alpha}\right)-\frac{1}{3-\alpha}\left(\frac{10}{3}-\frac{3}{4} \cdot 2^{3-\alpha}\right)-\frac{1}{12}\left(\frac{1}{2} \cdot 3^{2-\alpha}+\frac{1}{3-\alpha} \cdot 3^{3-\alpha}-4^{2-\alpha}\right) \\
& =-A(\alpha)-\frac{1}{3-\alpha} B(\alpha)-\frac{1}{12} D(\alpha),
\end{aligned}
$$

where $A(\alpha)=\frac{5}{3}-\frac{3}{8} \cdot 2^{2-\alpha}, \quad B(\alpha)=\frac{10}{3}-\frac{3}{4} \cdot 2^{3-\alpha}, \quad D(\alpha)=\frac{1}{2} \cdot 3^{2-\alpha}+\frac{1}{3-\alpha} \cdot 3^{3-\alpha}-4^{2-\alpha}$. It is easy to see that $A(\alpha)$ and $B(\alpha)$ are monotonically increasing for $1<\alpha<2$. Thus we have

and

$$
A(\alpha)>A(1)=\frac{11}{12}>0, \quad B(\alpha)>B(1)=\frac{1}{3}>0
$$

$$
\begin{aligned}
D(\alpha) & =\left(\frac{9}{2}+\frac{27}{3-\alpha}\right) 3^{-\alpha}-16 \cdot 4^{-\alpha}>\frac{9(9-\alpha)}{2(3-\alpha)} 3^{-\alpha}-16 \cdot 3^{-\alpha} \\
& =\left(\frac{27}{3-\alpha}-\frac{23}{2}\right) 3^{-\alpha}>D(1)=\frac{2}{3}>0,
\end{aligned}
$$

which implies that $d_{1}<0$ and

$$
\begin{aligned}
d_{3} & =\frac{1}{24(3-\alpha)}\left(284(7-\alpha) 2^{-\alpha}-351(9-\alpha) 3^{-\alpha}+480(3-\alpha) 4^{-\alpha}-40(5-\alpha)\right) \\
& =\frac{1}{24(3-\alpha)} F(\alpha),
\end{aligned}
$$

where

$$
F(\alpha)=284(7-\alpha) 2^{-\alpha}-351(9-\alpha) 3^{-\alpha}+480(3-\alpha) 4^{-\alpha}-40(5-\alpha)
$$

and

$$
F^{\prime}(\alpha)=284(-1-(7-\alpha) \ln 2) 2^{-\alpha}+351(1+(9-\alpha) \ln 3) 3^{-\alpha}+480(-1-(3-\alpha) \ln 4) 4^{-\alpha}+40 .
$$

By a direct calculation, one can find that $\tilde{\alpha}=1.2307$ is the unique zero point of $F^{\prime}(\alpha)=0$ for $\alpha \in(1,2)$. Thus $F^{\prime}(\alpha)>0$ for $(1, \tilde{\alpha})$ and $F^{\prime}(\alpha)<0$ for $(\tilde{\alpha}, 2)$, which implies that $\tilde{\alpha}$ is the only maximum of $F(\alpha)$. Since $F(\tilde{\alpha})=-3.8948<0$, we have $F(\alpha)<0$ for all $\alpha \in(1,2)$. Hence we get $d_{3}<0$. For $k \geq 5$, we can prove $d_{1}<0, d_{3}<0$ in a similar way.

For (52), we first prove $d_{2}$ for the different $k$. When $k=4$, we have

$$
d_{2}=\frac{1}{8(3-\alpha)}\left(-60(7-\alpha) 2^{-\alpha}+45(9-\alpha) 3^{-\alpha}-32(3-\alpha) 4^{-\alpha}+20(5-\alpha)\right)=\frac{1}{8(3-\alpha)} H(\alpha),
$$


where

$$
H(\alpha)=-60(7-\alpha) 2^{-\alpha}+45(9-\alpha) 3^{-\alpha}-32(3-\alpha) 4^{-\alpha}+20(5-\alpha) .
$$

Furthermore, we have

$$
H^{\prime}(\alpha)=-60(-1-(7-\alpha) \ln 2) 2^{-\alpha}+45(-1-(9-\alpha) \ln 3) 3^{-\alpha}-32(-1-(3-\alpha) \ln 4) 4^{-\alpha}-20
$$

and

$$
\begin{aligned}
H^{\prime \prime}(\alpha) & =-60 \ln 2(2+(7-\alpha) \ln 2) 2^{-\alpha}-45 \ln 3(-2-(9-\alpha) \ln 3) 3^{-\alpha}-32 \ln 4(2+(3-\alpha) \ln 4) 4^{-\alpha} \\
& =\bar{A}(\alpha) \cdot 2^{-\alpha}+\bar{B}(\alpha) \cdot 4^{-\alpha}-\bar{D}(\alpha) \cdot 3^{-\alpha}
\end{aligned}
$$

where

$$
\begin{aligned}
& \bar{A}(\alpha)=-60 \ln 2(2+(7-\alpha) \ln 2), \\
& \bar{B}(\alpha)=-32 \ln 4(2+(3-\alpha) \ln 4), \\
& \bar{D}(\alpha)=-45 \ln 3(2+(9-\alpha) \ln 3) .
\end{aligned}
$$

By the simple calculations, we get

$$
\bar{A}(\alpha)<0, \bar{B}(\alpha)<0, \bar{D}(\alpha)<0 \quad \text { for } 1<\alpha<2 .
$$

Note that

$$
\begin{aligned}
& H^{\prime \prime}(\alpha)=\bar{A}(\alpha) \cdot 2^{-\alpha}+\bar{B}(\alpha) \cdot 4^{-\alpha}-\bar{D}(\alpha) \cdot 3^{-\alpha}=\frac{\bar{A}(\alpha)}{4} \cdot 2^{2-\alpha}+\frac{\bar{B}(\alpha)}{16} \cdot 4^{2-\alpha}-\frac{\bar{D}(\alpha)}{9} \cdot 3^{2-\alpha} \\
& =\frac{\bar{A}(\alpha)}{4} 3^{2-\alpha}\left(1-\frac{1}{3}\right)^{2-\alpha}+\frac{\bar{B}(\alpha)}{16} 3^{2-\alpha}\left(1+\frac{1}{3}\right)^{2-\alpha}-\frac{\bar{D}(\alpha)}{9} 3^{2-\alpha} \\
& =\frac{\bar{A}(\alpha)}{4} 3^{2-\alpha}\left[1-\frac{2-\alpha}{3}+\frac{(2-\alpha)(1-\alpha)}{2 !}\left(-\frac{1}{3}\right)^{2}+\frac{(2-\alpha)(1-\alpha)(-\alpha)}{3 !}\left(-\frac{1}{3}\right)^{3}+\cdots\right] \\
& +\frac{\bar{B}(\alpha)}{16} 3^{2-\alpha}\left[1+\frac{2-\alpha}{3}+\frac{(2-\alpha)(1-\alpha)}{2 !}\left(\frac{1}{3}\right)^{2}+\frac{(2-\alpha)(1-\alpha)(-\alpha)}{3 !}\left(\frac{1}{3}\right)^{3}+\cdots\right]-\frac{\bar{D}(\alpha)}{9} 3^{2-\alpha} \\
& =3^{2-\alpha}\left[\frac{\bar{A}(\alpha)}{4}+\frac{\bar{B}(\alpha)}{16}-\frac{\bar{D}(\alpha)}{9}+\left(-\frac{\bar{A}(\alpha)}{4}+\frac{\bar{B}(\alpha)}{16}\right) \frac{2-\alpha}{3}+\frac{(2-\alpha)(1-\alpha)}{2 !}\left(\frac{\bar{A}(\alpha)}{4}+\frac{\bar{B}(\alpha)}{16}+\frac{(-\alpha)}{3 \cdot 3}\left(-\frac{\bar{A}(\alpha)}{4}+\right.\right.\right. \\
& \left.\left.\left.+\frac{\bar{B}(\alpha)}{16}\right)\right)\left(\frac{1}{3}\right)^{2}+\frac{(2-\alpha)(1-\alpha)(-\alpha)(-\alpha-1)}{4 !}\left(\frac{\bar{A}(\alpha)}{4}+\frac{\bar{B}(\alpha)}{16}+\frac{(-\alpha-2)}{5 \cdot 3}\left(-\frac{\bar{A}(\alpha)}{4}+\frac{\bar{B}(\alpha)}{16}\right)\right)\left(\frac{1}{3}\right)^{4}+\cdots\right] \\
& =3^{2-\alpha}\left[\frac{(12 \bar{A}(\alpha)+15 \bar{B}(\alpha)-16(\alpha))+3(4 \bar{A}(\alpha)-\bar{B}(\alpha)) \alpha}{144}\right. \\
& \left.+\sum_{k=1}^{\infty} \frac{(2-\alpha)(1-\alpha)(-\alpha)(-\alpha-(2 k-3))}{(2 k) !}\left(\frac{\bar{A}(\alpha)}{4}+\frac{\bar{B}(\alpha)}{16}+\frac{(-\alpha-(2 k-2))}{(2 k+1) \cdot 3}\left(-\frac{\bar{A}(\alpha)}{4}+\frac{\bar{B}(\alpha)}{16}\right)\right)\left(\frac{1}{3}\right)^{2 k}\right] \\
& =3^{2-\alpha}\left[\frac{(12 \bar{A}(\alpha)+15 \bar{B}(\alpha)-16 \bar{D}(\alpha))+3(4 \bar{A}(\alpha)-\bar{B}(\alpha)) \alpha}{144}\right. \\
& \left.+\sum_{k=1}^{\infty} \frac{-(2-\alpha)(\alpha+(2 k-3))(4(8 k+1+\alpha) \bar{A}(\alpha)+(4 k-\alpha+5) \bar{B}(\alpha))}{48(2 k+1) !}\left(\frac{1}{3}\right)^{2 k}\right] \\
& =3^{2-\alpha}\left(q(\alpha)+S_{\alpha}\right)
\end{aligned}
$$

Here

$$
\begin{aligned}
q(\alpha)= & (12 \bar{A}(\alpha)+15 \bar{B}(\alpha)-16 \bar{D}(\alpha))+3(4 \bar{A}(\alpha)-\bar{B}(\alpha)) \alpha \\
= & 336(\ln 2)^{2} \alpha^{2}+\left(-1248(\ln 2)^{2}-720(\ln 3)^{2}-1056(\ln 2)\right) \alpha \\
& -10800(\ln 2)^{2}+6480(\ln 3)^{2}+1440(\ln 3)-3360(\ln 2),
\end{aligned}
$$


and

$$
S_{\alpha}=\sum_{k=1}^{\infty} V_{\alpha}(k)\left(\frac{1}{3}\right)^{2 k}
$$

where

$$
V_{\alpha}(k)=\frac{-(2-\alpha)(\alpha+(2 k-3))(4(8 k+1+\alpha) A+(4 k-\alpha+5) B)}{48(2 k+1) !} .
$$

By the tedious calculations, we see that $q(\alpha)$ is decreasing for $1<\alpha<2$. Since $q(1)=-153.9759<0$, we therefore get $q(\alpha)<q(1)=-153.9759$ for all $1<\alpha<2$. Noting that

$$
V_{\alpha}(k)<V_{\alpha}(1)=\frac{-(2-\alpha)(\alpha-1)(4(9+\alpha) \bar{A}(\alpha)+(9-\alpha) \bar{B}(\alpha))}{288},
$$

we have

$$
S_{\alpha}<\sum_{k=1}^{\infty} V_{\alpha}(1) \cdot\left(\frac{1}{3}\right)^{2 k}=V_{\alpha}(1) \frac{\frac{1}{9}}{1-\frac{1}{9}}=\frac{1}{8} V_{\alpha}(1) .
$$

By mathematical analysis, we find that $\overline{\bar{\alpha}}=1.4900$ is the only maximum point of $V_{\alpha}(1)$. Since $V_{\overline{\bar{\alpha}}}(1)=$ 9.9948, we have

$$
S_{\alpha}<\frac{1}{8} V_{\overline{\bar{\alpha}}}(1)=1.2494 .
$$

Thus we conclude that $H^{\prime \prime}(\alpha)<0$ for $1<\alpha<2$ which implies that $H^{\prime}(\alpha)$ is decreasing for $1<\alpha<2$. Hence we get $H^{\prime}(\alpha)>H^{\prime}(2)=8.3072>0$ for $1<\alpha<2$, which implies that $H(\alpha)$ is increasing on $1<\alpha<2$. Since $H(1)=4$, we get $H(\alpha)>H(1)=4>0$ which implies that $d_{2}>0$. Similarly we may show $d_{2}>0$ for $k \geq 5$.

Now we consider $d_{j}, j>4$. When $k \geq 5$, we have

$$
\begin{aligned}
w_{l}^{(\alpha)}= & b_{l}^{(\alpha)}+e_{l}^{(\alpha)}-e_{l-1}^{(\alpha)} \\
= & {\left[\frac{1}{3-\alpha}\left((l+1)^{3-\alpha}-l^{3-\alpha}\right)+\frac{1}{2}\left((l+1)^{2-\alpha}-l^{2-\alpha}\right)\right] } \\
& -\left[\frac{1}{3-\alpha}\left(l^{3-\alpha}-(l-1)^{3-\alpha}\right)+\frac{1}{2}\left(l^{2-\alpha}-(l-1)^{2-\alpha}\right)\right] \\
= & h(l+1)-2 h(l)+h(l-1),
\end{aligned}
$$

where

$$
h(x)=\frac{1}{3-\alpha} x^{3-\alpha}+\frac{1}{2} x^{2-\alpha} \quad \text { for } x \geq 5 .
$$

Thus we get

$$
w_{j+1}^{(\alpha)}-2 w_{j}^{(\alpha)}+w_{j-1}^{(\alpha)}=h(j+2)-4 h(j+1)+6 h(j)-4 h(j-1)+h(j+2)=h^{(4)}(\xi),
$$

where $\xi \in(j-2, j+2), 4<j \leq k-2$.

Noting that

$$
\begin{aligned}
& h^{\prime}(x)=x^{2-\alpha}+\frac{1}{2}(2-\alpha) x^{1-\alpha}>0, \\
& h^{\prime \prime}(x)=(2-\alpha) x^{1-\alpha}+\frac{1}{2}(2-\alpha)(1-\alpha) x^{-\alpha}=(2-\alpha) x^{-\alpha}\left[x-\frac{1}{2}(\alpha-1)\right]>0, \\
& h^{(3)}(x)=(2-\alpha)(\alpha-1) x^{-\alpha-1}\left[\frac{\alpha}{2}-x\right]<0, \\
& h^{(4)}(x)=(2-\alpha)(\alpha-1) \alpha x^{-\alpha-2}\left(x-\frac{\alpha+1}{2}\right)>0,
\end{aligned}
$$


we obtain $w_{j+1}^{(\alpha)}+w_{j-1}^{(\alpha)}>2 w_{j}^{(\alpha)}$. Based on the above results, we see that $w^{(\alpha)}(x)$ is a convex function. We therefore obtain, with $4<j \leq k-2$,

$$
d_{j}=\frac{1}{12}\left(w_{j-3}^{(\alpha)}+w_{j+1}^{(\alpha)}\right)+\frac{2}{3}\left(w_{j-2}^{(\alpha)}+w_{j}^{(\alpha)}\right)-\frac{3}{2} w_{j-1}^{(\alpha)}>\frac{1}{12} \cdot 2 w_{j-1}^{(\alpha)}+\frac{2}{3} \cdot 2 w_{j-1}^{(\alpha)}-\frac{3}{2} w_{j-1}^{(\alpha)}=0 .
$$

For $j=k-3, k-2, k-1, k$, we have

$$
\begin{aligned}
d_{k-3}= & \frac{1}{12} b_{k-6}^{(\alpha)}+\frac{2}{3} b_{k-5}^{(\alpha)}-\frac{5}{2} b_{k-4}^{(\alpha)}+\frac{2}{3} b_{k-3}^{(\alpha)}+\frac{1}{12} b_{k-1}^{(\alpha)} \\
& -\frac{1}{12} e_{k-7}^{(\alpha)}-\frac{7}{12} e_{k-6}^{(\alpha)}+\frac{19}{6} e_{k-5}^{(\alpha)}-\frac{19}{6} e_{k-4}^{(\alpha)}+\frac{2}{3} e_{k-3}^{(\alpha)}-\frac{1}{12} e_{k-2}^{(\alpha)}, \\
d_{k-2}= & \frac{1}{12} b_{k-5}^{(\alpha)}+\frac{2}{3} b_{k-4}^{(\alpha)}-\frac{3}{2} b_{k-3}^{(\alpha)}+b_{k-2}^{(\alpha)}-\frac{1}{4} b_{k-1}^{(\alpha)} \\
& -\frac{1}{12} e_{k-6}^{(\alpha)}-\frac{7}{12} e_{k-5}^{(\alpha)}+\frac{13}{6} e_{k-4}^{(\alpha)}-\frac{5}{2} e_{k-3}^{(\alpha)}+\frac{5}{4} e_{k-2}^{(\alpha)}, \\
d_{k-1}= & \frac{1}{12} b_{k-4}^{(\alpha)}+\frac{2}{3} b_{k-3}^{(\alpha)}-2 b_{k-2}^{(\alpha)}+\frac{5}{4} b_{k-1}^{(\alpha)}-\frac{1}{12} e_{k-5}^{(\alpha)}-\frac{7}{12} e_{k-4}^{(\alpha)}+\frac{8}{3} e_{k-3}^{(\alpha)}-\frac{13}{4} e_{k-2}^{(\alpha)}, \\
d_{k}= & \frac{1}{12} b_{k-3}^{(\alpha)}+b_{k-2}^{(\alpha)}-\frac{13}{12} b_{k-1}^{(\alpha)}-\frac{1}{12} e_{k-4}^{(\alpha)}-\frac{11}{12} e_{k-3}^{(\alpha)}+\frac{25}{12} e_{k-2}^{(\alpha)} .
\end{aligned}
$$

Similarly, we can show $d_{l}>0$ for $l=k-3, k-2, k-1, k$. Hence (52) holds.

We now consider (53). For $k=4$, by mathematical analysis, there exists a unique point $\alpha_{1}=1.8047$, such that

$$
-\frac{3}{2} 2^{2-\alpha}+\frac{25}{24} 3^{2-\alpha}-\frac{13}{12} 4^{2-\alpha}+\frac{5}{6(3-\alpha)}-\frac{3}{3-\alpha} 2^{3-\alpha}+\frac{25}{12(3-\alpha)} 3^{3-\alpha}=0, \quad \alpha \in(1,2),
$$

which implies that $d_{4}>0$ if $\alpha \in\left(1, \alpha_{1}\right)$.

For $k=5$, it is not difficult to show that

$$
\begin{aligned}
d_{4} & =\frac{1}{12} b_{1}^{(\alpha)}+\frac{2}{3} b_{2}^{(\alpha)}-2 b_{3}^{(\alpha)}+\frac{5}{4} b_{4}^{(\alpha)}-\frac{1}{12} e_{0}^{(\alpha)}-\frac{7}{12} e_{1}^{(\alpha)}+\frac{8}{3} e_{2}^{(\alpha)}-\frac{13}{4} e_{3}^{(\alpha)} \\
& =\frac{5-\alpha}{4(3-\alpha)}-\frac{13(7-\alpha)}{2(3-\alpha)} 2^{-\alpha}+\frac{213(9-\alpha)}{8(3-\alpha)} 3^{-\alpha}-\frac{26(11-\alpha)}{3-\alpha} 4^{-\alpha}+\frac{125}{4} 5^{-\alpha} .
\end{aligned}
$$

By tedious calculations, one can obtain that $d_{4} \in(-0.0417,0)$ which implies that $d_{4}<0$.

For $k=6$, we have

$$
\begin{aligned}
d_{4}= & -\frac{11}{8} 2^{2-\alpha}+\frac{1}{4}-\frac{11}{4(3-\alpha)} 2^{3-\alpha}+\frac{1}{2(3-\alpha)}+\frac{7}{3} 3^{2-\alpha}+\frac{14}{3(3-\alpha)} 3^{3-\alpha} \\
& -\frac{15}{8} 4^{2-\alpha}-\frac{15}{4(3-\alpha)} 4^{3-\alpha}+\frac{5}{8} 5^{2-\alpha}+\frac{5}{4(3-\alpha)} 5^{3-\alpha}-\frac{1}{4} 6^{2-\alpha} .
\end{aligned}
$$

By mathematics analysis, there exists a unique $\alpha_{2}=1.6628$, such that $d_{4}\left(\alpha_{2}\right)=0$, which implies that $d_{4}>0$ if $\alpha \in\left(1, \alpha_{2}\right)$.

For $k=7$, we have

$$
d_{4}=\frac{1}{12} b_{1}^{(\alpha)}+\frac{2}{3} b_{2}^{(\alpha)}-\frac{3}{2} b_{3}^{(\alpha)}+\frac{2}{3} b_{4}^{(\alpha)}+\frac{1}{12} b_{5}^{(\alpha)}-\frac{1}{12} e_{0}^{(\alpha)}-\frac{7}{12} e_{1}^{(\alpha)}+\frac{13}{6} e_{2}^{(\alpha)}-\frac{13}{6} e_{3}^{(\alpha)}+\frac{2}{3} e_{4}^{(\alpha)}-\frac{1}{12} e_{5}^{(\alpha)} .
$$

By calculation, there exists a unique $\alpha_{3}=1.5878$, such that $d_{4}\left(\alpha_{3}\right)=0$, which implies that $d_{4}>0$ if $\alpha \in\left(1, \alpha_{3}\right)$.

For $k>7$, one can easily show that $d_{4}>0$ for all $\alpha \in(1,2)$. Together these estimates we conclude that $d_{4}>0$ if $\alpha \in(1,1.5878)$.

Finally (54) follows directly from equations (26).

Together these estimates complete the proof of Lemma 3.2. 


\section{Numerical simulations}

In this section, we will give some numerical examples to illustrate our approximate schemes.

\subsection{Three test examples}

In this section, we will consider three numerical examples to test the approximate scheme (24).

Example 4.1. Let $f(t)=t^{4+\alpha}, 1<\alpha<2$, we have

$$
{ }_{0}^{C} D_{t}^{\alpha} f(t)=\frac{\Gamma(5+\alpha)}{24} t^{4} .
$$

Let $0=t_{0}<t_{1}<t_{2}<\cdots<t_{N}=1$ be a partition on $[0,1]$ and $\Delta t$ the step size. We will consider the approximation scheme (24) at $t_{N}=1$. Choosing the different step size $\Delta t=\frac{1}{K}$ with $K=10 \times 2^{l}, l=$ $0,1, \ldots, 7$, in Table 1 , we obtain the computational errors and numerical convergence orders with the different $\alpha=1.1,1.5,1.9$ for the approximation at $t_{N}=1$ in (24). From the results presented in Table 1, we observe that the convergence order is $\Delta t^{4-\alpha}$ as we expected.

\begin{tabular}{cccccccccc}
$\alpha$ & $K=10$ & $K=20$ & $K=40$ & $K=80$ & $K=160$ & $K=320$ & $K=640$ & $K=1280$ & order \\
\hline 1.1 & $1.64 \mathrm{e}-2$ & $2.40 \mathrm{e}-3$ & $3.38 \mathrm{e}-4$ & $4.68 \mathrm{e}-5$ & $6.42 \mathrm{e}-6$ & $8.76 \mathrm{e}-7$ & $1.19 \mathrm{e}-7$ & $1.62 \mathrm{e}-8$ & 2.88 \\
1.5 & $1.32 \mathrm{e}-1$ & $2.61 \mathrm{e}-2$ & $4.90 \mathrm{e}-3$ & $8.90 \mathrm{e}-4$ & $1.60 \mathrm{e}-4$ & $2.86 \mathrm{e}-5$ & $5.09 \mathrm{e}-6$ & $9.03 \mathrm{e}-7$ & 2.49 \\
1.9 & $8.33 \mathrm{e}-1$ & $2.19 \mathrm{e}-1$ & $5.43 \mathrm{e}-2$ & $1.30 \mathrm{e}-2$ & $3.10 \mathrm{e}-3$ & $7.26 \mathrm{e}-4$ & $1.70 \mathrm{e}-4$ & $3.97 \mathrm{e}-5$ & 2.10 \\
\hline
\end{tabular}

Table 1: The convergence orders with the different $\alpha \in(1,2)$ at $t_{N}=1$ in (24) in Example 4.1

Example 4.2. Let $f(t)=e^{2 t}-2 t-2 t^{2}-\frac{4}{3} t^{3}-\frac{2}{3} t^{4}, 1<\alpha<2$. We have

$$
{ }_{0}^{C} D_{t}^{\alpha} f(t)=2 t^{1-\alpha} E_{1,2-\alpha}(2 t)-\sum_{n=1}^{4} \frac{2^{n} t^{n-\alpha}}{\Gamma(n+1-\alpha)},
$$

where $E_{\alpha, \beta}(z)$ is the Mittag-Leffler function with two parameters defined by

$$
E_{\alpha, \beta}(z)=\sum_{n=0}^{\infty} \frac{z^{n}}{\Gamma(\alpha n+\beta)}
$$

\begin{tabular}{cccccccccc}
$\alpha$ & $K=10$ & $K=20$ & $K=40$ & $K=80$ & $K=160$ & $K=320$ & $K=640$ & $K=1280$ & order \\
\hline 1.1 & $1.08 \mathrm{e}-2$ & $1.70 \mathrm{e}-3$ & $2.43 \mathrm{e}-4$ & $3.42 \mathrm{e}-5$ & $4.73 \mathrm{e}-6$ & $6.49 \mathrm{e}-7$ & $8.86 \mathrm{e}-8$ & $1.22 \mathrm{e}-8$ & 2.86 \\
1.5 & $5.72 \mathrm{e}-2$ & $1.17 \mathrm{e}-2$ & $2.20 \mathrm{e}-3$ & $4.14 \mathrm{e}-4$ & $7.50 \mathrm{e}-5$ & $1.34 \mathrm{e}-5$ & $2.39 \mathrm{e}-6$ & $4.25 \mathrm{e}-7$ & 2.49 \\
1.9 & $2.51 \mathrm{e}-1$ & $6.72 \mathrm{e}-2$ & $1.68 \mathrm{e}-2$ & $4.10 \mathrm{e}-3$ & $9.63 \mathrm{e}-4$ & $2.27 \mathrm{e}-4$ & $5.31 \mathrm{e}-5$ & $1.24 \mathrm{e}-5$ & 2.10 \\
\hline
\end{tabular}

Table 2: The convergence orders with the different $\alpha \in(1,2)$ at $t_{N}=1$ in (24) in Example 4.2

We use the same notations as in Example 4.1. We also observe that, in Table 2, the convergence order of the approximate scheme (24) is $O\left(\Delta t^{4-\alpha}\right)$ as we expected.

The functions of the above two examples all satisfy the condition $f^{\prime \prime \prime}\left(t_{0}\right)=0$. In the following example, we shall consider a function $f$ which does not satisfy the condition $f^{\prime \prime \prime}\left(t_{0}\right)=0$. 
Example 4.3. Let $f(t)=t^{3}, 1<\alpha<2$. We have

\begin{tabular}{cccccccccc}
\multicolumn{10}{c}{${ }_{0}^{C} D_{t}^{\alpha} f(t)=\frac{\Gamma(4)}{\Gamma(4-\alpha)} t^{3-\alpha}}$, \\
\\
& $K=10$ & $K=20$ & $K=40$ & $K=80$ & $K=160$ & $K=320$ & $K=640$ & $K=1280$ & order \\
\hline 1.1 & $3.88 \mathrm{e}-5$ & $1.04 \mathrm{e}-5$ & $2.79 \mathrm{e}-6$ & $7.46 \mathrm{e}-7$ & $1.99 \mathrm{e}-7$ & $5.36 \mathrm{e}-8$ & $1.43 \mathrm{e}-8$ & $3.85 \mathrm{e}-9$ & 1.85 \\
1.5 & $1.21 \mathrm{e}-4$ & $3.24 \mathrm{e}-5$ & $8.69 \mathrm{e}-6$ & $2.33 \mathrm{e}-6$ & $6.24 \mathrm{e}-7$ & $1.67 \mathrm{e}-7$ & $4.48 \mathrm{e}-8$ & $1.19 \mathrm{e}-8$ & 1.44 \\
1.9 & $4.22 \mathrm{e}-5$ & $1.13 \mathrm{e}-5$ & $3.03 \mathrm{e}-6$ & $8.12 \mathrm{e}-7$ & $2.18 \mathrm{e}-7$ & $5.83 \mathrm{e}-8$ & $1.56 \mathrm{e}-8$ & $4.18 \mathrm{e}-9$ & 1.05 \\
\hline
\end{tabular}

Table 3: The convergence orders with the different $\alpha \in(1,2)$ at $t_{N}=1$ in (24) in Example 4.3

Using the same notations as in Example 4.1, we can observe from Table 3, although the errors are relatively small for the different values of $\alpha$, the convergence order indeed can not reach $O\left(\Delta t^{4-\alpha}\right), 1<\alpha<2$ which is consistent with Theorem 2.4. In other words, the approximation scheme (24) has the convergence order $O\left(\Delta t^{4-\alpha}\right), 1<\alpha<2$ only for the function $f$ with $f^{\prime \prime \prime}\left(t_{0}\right)=0$.

\subsection{A high-order numerical method for solving time fractional diffusion wave equation}

In this subsection, we will use the numerical method (47)-(49) to solve the time fractional diffusion wave equation (2)-(4).

Example 4.4. In (2)-(4), we choose $F(x, t)=\frac{\Gamma(4+1)}{\Gamma(4+1-\alpha)} t^{4-\alpha}\left(x-x^{2}\right)+2 t^{4}, \phi(t)=0, \varphi(t)=0, u_{0}(x)=$ $0, u_{1}(x)=0$.

The exact solution is $u(x, t)=t^{4} x(1-x), 0<x<1,0<t<T$ with $T=1$.

To verify the temporal numerical accuracy, we will choose $M=2^{6}$ sufficiently big such that the spatial error is negligible as compared to the temporal error. The numerical results are computed under the different time step sizes $\Delta t=\frac{1}{K}$ using the numerical method (47)-(49) at $t_{N}=1$. The solution errors in discrete $L^{2}$ norm and numerical convergence orders for $T=1$ with the different $\alpha=1.1,1.5,1.9$ are tabulated in Table 3.

The data in Table 3 show that the numerical accuracy of the difference scheme (47)-(49) achieves $4-\alpha$ in temporal direction.

\begin{tabular}{ccccccc}
$\alpha$ & $K=2^{4}$ & $K=2^{5}$ & $K=2^{6}$ & $K=2^{7}$ & $K=2^{8}$ & order \\
\hline 1.1 & $1.60 \mathrm{e}-5$ & $2.25 \mathrm{e}-6$ & $3.10 \mathrm{e}-7$ & $4.00 \mathrm{e}-8$ & $1.00 \mathrm{e}-8$ & 2.88 \\
1.5 & $1.35 \mathrm{e}-4$ & $2.26 \mathrm{e}-5$ & $3.80 \mathrm{e}-6$ & $7.00 \mathrm{e}-7$ & $1.00 \mathrm{e}-7$ & 2.58 \\
1.9 & $8.37 \mathrm{e}-4$ & $2.24 \mathrm{e}-4$ & $5.30 \mathrm{e}-5$ & $1.23 \mathrm{e}-5$ & $2.90 \mathrm{e}-6$ & 2.11 \\
\hline
\end{tabular}

Table 4: Time convergence orders with the different $\alpha \in(1,2)$ at $T=1$ in (47)-(49) in Example 4.4

As an exceptional application, in order to further verify the ability of the new formula to solve the fractional diffusion equations, we consider the Caputo-type time fractional diffusion wave equation, which have non-zero boundary conditions.

Example 4.5. In (2)-(4), we choose $F(x, t)=e^{x} t^{4}\left(\frac{\Gamma(5+\alpha)}{24}-t^{\alpha}\right), \phi(t)=t^{4+\alpha}, \varphi(t)=e t^{4+\alpha}, u_{0}(x)=$ $0, u_{1}(x)=0$.

The exact solution is $u(x, t)=e^{x} t^{4+\alpha}, 0<x<1,0<t<1$. 
To obtain the convergence order with respect to time, similar to Example 4.4, we choose the space step size $h=1 / 2^{6}$ and the various time step sizes $\Delta t=1 / 2^{3}, 1 / 2^{4}, 1 / 2^{5}, 1 / 2^{6}, 1 / 2^{7}$ respectively. The numerical errors and convergence orders with the different temporal step sizes are listed in Table 5. From Table 5, we observe that the convergence order of (47)-(49) in temporal direction is $O\left(\Delta t^{4-\alpha}\right)$ as we expected.

\begin{tabular}{cccc}
$\alpha$ & $\Delta t$ & Error & order \\
\hline 1.1 & $1 / 2^{3}$ & $4.50 \mathrm{e}-3$ & - \\
& $1 / 2^{4}$ & $6.00 \mathrm{e}-4$ & 2.6808 \\
& $1 / 2^{5}$ & $1.00 \mathrm{e}-4$ & 2.8133 \\
& $1 / 2^{6}$ & $1.42 \mathrm{e}-5$ & 2.8119 \\
1.5 & $1 / 2^{3}$ & $2.69 \mathrm{e}-2$ & - \\
& $1 / 2^{4}$ & $6.00 \mathrm{e}-3$ & 2.1550 \\
& $1 / 2^{5}$ & $1.10 \mathrm{e}-3$ & 2.4313 \\
& $1 / 2^{6}$ & $2.00 \mathrm{e}-4$ & 2.4521 \\
1.9 & $1 / 2^{4}$ & $3.49 \mathrm{e}-2$ & - \\
& $1 / 2^{5}$ & $8.90 \mathrm{e}-3$ & 1.9731 \\
& $1 / 2^{6}$ & $2.10 \mathrm{e}-3$ & 2.0492 \\
& $1 / 2^{7}$ & $5.00 \mathrm{e}-4$ & 2.0740 \\
\hline
\end{tabular}

Table 5: Time convergence orders with the different $\alpha \in(1,2)$ at $T=1$ in (47)-(49) in Example 4.5

\section{Conclusion}

In this paper, a new finite difference scheme to approximate the Caputo fractional derivative with the convergence order $O\left(\Delta t^{4-\alpha}\right), 1<\alpha<2$ was constructed when $f^{\prime \prime \prime}\left(t_{0}\right)=0$. This new scheme was obtained by using the piecewise interpolation polynomial to approximate the integrand $g(t)=f^{\prime}(t)$, which can also be seen as an improvement to the $L 2$ and $L 2 C$ schemes discussed in the Induction section. The computational and storage cost of the proposed numerical scheme to approximate the Caputo fractional derivative in this paper does not increase much compared with the lower convergence order numerical scheme available in literature. The properties of the weights and the truncation error in the approximate scheme are analyzed. Three numerical examples are carried out to effectively confirm the computational validity and numerical accuracy of the proposed numerical scheme for approximating the Caputo fractional derivative. In addition, we also consider two numerical examples for solving time fractional diffusion wave equations on a bounded spatial domain by using the proposed new scheme for approximating Caputo fractional derivative.

From the numerical results, it is easy to see that the new scheme developed in this paper to approximate the Caputo fractional derivative has the convergence order $O\left(\Delta t^{4-\alpha}\right), 1<\alpha<2$ when $f^{\prime \prime \prime}\left(t_{0}\right)=0$, which is better than $L 2$ and $L 2 C$ schemes. This new difference scheme can be applied to many other equations involving the Caputo fractional derivative. In our future work, we will consider the stability and error estimates for the proposed numerical method in this paper for solving fractional diffusion wave equation.

\section{References}

[1] O. P. Agrawal, Solution for a fractional diffusion-wave equation defined in a bounded domain, Nonlinear Dynamics, 29(2002), pp. 145155.

[2] O. P. Agrawal, Response of a diffusion-wave system subjected to deterministic and stochastic fields, Z. Angew. Math. Mech., 83 (2003), pp. 265-274.

[3] J.X. Cao, C.P. Li, and Y. Q. Chen, High-order approximation to Caputo derivatives and Caputo-type advection-diffusion equations(II), Fractional calculus and Applied analysis, 18 (2015), pp. 735-761.

[4] M.H.Chen, W.H.Deng, A second-order accurate numerical method for the space-time tempered fractional diffusion-wave equation, Applied Mathematics Letters, 68 (2017), pp. 87-93. 
[5] A. Chen and C.P. Li, Numerical solution of fractional diffusion-wave equation, Numerical Functional Analysis and Optimization 37 (2016), pp. 19-39.

[6] M. Dehghan, M. Abbaszadeh, and W. Deng, Fourth-order numerical method for the space-time tempered fractional diffusion-wave equation, Applied Mathematics Letters, 73 (2017) 120-127.

[7] R. Du, W.R. Cao, and Z.Z. Sun, A compact difference scheme for the fractional diffusion-wave equation, Applied Mathematical Modelling, 34 (2010), pp. 2998-3007.

[8] N. J. Ford, K. Pal and Y. Yan, An algorithm for the numerical solution of space-fractional partial differential equations, Computational Methods in Applied Mathematics, 15 (2015), pp. 497-514.

[9] Y. Fujita, Cauchy problems of fractional order and stable processes, Japan J. Appl. Math., 7 (1990), pp. 459-476.

[10] Y. Fujita, Integro differential equation which interpolates the heat equation and the wave equation, Osaka J. Math., 27 (1990), pp. 309-321.

[11] Y. Fujita, Integro differential equation which interpolates the heat equation and the wave equation II, Osaka J. Math., 27 (1990), pp. 797-804.

[12] G. H. Gao, Z. Z. Sun, and H. W. Zhang, A new fractional numerical differentiation formula to approximate the Caputo fractional derivative and its applications, J. Comput. Phys., 259 (2014), pp. 33-50.

[13] J.F. Huang, Y.F. Tang, L. Vázquez, and J.Y. Yang, Two finite difference schemes for time fractional diffusion-wave equation, Numerical Algorithms, 64 (2013), pp. 707-720.

[14] C.P. Li and M. Cai, High-order approximation to Caputo derivatives and Caputo-type advection-diffusion equations: Revisited, Numerical functional analysis and optimization, 38 (2017), pp. 861-890.

[15] H.F. Li, J.X. Cao, and C.P. Li, High-order approximation to Caputo derivatives and Caputo-type advection-diffusion equations(III), Journal of computational and applied mathematics, 299 (2016), pp. 159-175.

[16] C. Li and A. Chen, Numerical methods for fractional partial differential equations, International Journal of Computer Mathematics, 95 (2017), pp. 1048-1099.

[17] C.P. Li, R.F. Wu, and H.F. Ding, High-order approximations to Caputo derivatives and Caputo-type advection-diffusion equations, Communications in Applied and Industrial Mathematics, 6 (2014), pp. 1-33.

[18] Z. Li, Y. Yan and N. J. Ford, Error estimates of a high order numerical method for solving linear fractional differential equation, Applied Numerical Mathematics, 114 (2017), pp. 201-220.

[19] C. Lubich, Discretized fractional calculus, SIAM Journal on Mathematical Analysis, 17 (1986), pp. 704-719.

[20] Y. Luchko, Fractional wave equation and damped waves, J. Math. Phys., 54 (2013), 031505.

[21] Y. Luchko, Wave-diffusion dualism of the neutral-fractional processes, J. Comput. Phys., 293 (2015), pp. 40-52.

[22] Y. Luchko, F. Mainardi, and Y. Povstenko, Propagation speed of the maximum of the fundamental solution to the fractional diffusion-wave equation, Comput. Math. Appl., 66 (2013), pp. 774-784.

[23] W.H. Luo, C.P. Li, T.Z. Huang, X.M Gu, and G.C.Wu, A high-order accurate numerical scheme for the Caputo derivative with an application to fractional diffusion problems, Numerical functional analysis and optimization, 39 (2018), pp. 600622 .

[24] V. Lynch, B. Carreras, D. del Castillo-Negrete, K. Ferreira-Mejias, and H. Hicks, Wright function as scale-invariant solutions of the diffusion-wave equation, Journal of Computational Physics 192 (2003), pp. 406-421.

[25] F. Mainardi, Fractional Calculus and Waves in Linear Viscoelasticity: An Introduction to Mathematical Models, Imperial College Press, London, 2010.

[26] W. R. Schneider and W.Wyss, Fractional diusion and wave equations, J.Math. Phys., 30 (1989), pp. 134-144.

27 E. Sousa and C. Li, A weighted finite difference method for the fractional diffusion equation based on the Riemann-Liouville derivative, Applied Numerical Mathematics, 90 (2015), pp. 22-37.

[28] Z.Z.Sun and G.H.Gao, Finite Difference Method for Fractional Differential Equations, Science Press, Beijing, 2015 (in Chinese).

[29] Z.Z. Sun and X.N. Wu, A fully discrete difference scheme for a diffusion-wave system, Applied Numerical Mathematics, 56 (2006), pp. 193-209.

[30] W.Y. Tian, H. Zhou, and W.H. Deng, A class of second order difference approximations for solving space fractional diffusion equations, Mathematics of Computation, 84 (2015), pp. 1703-1727.

[31] Z.B. Wang and S. Vong, Compact difference schemes for the modified anomalous fractional subdiffusion equation and the fractional diffusion-wave equation, Journal of Computational Physics, 277 (2014), pp. 1-15.

[32] L. Wei, Analysis of a new finite difference/local discontinuous Galerkin method for the fractional diffusion-wave equation, Appl. Math. Comput., 304 (2017), pp. 180-189.

[33] Y. Yan, K. Pal and N. J. Ford, Higher order numerical methods for solving fractional differential equations, BIT Numer. Math., 54 (2014), pp. 555-584

[34] J.Y. Yang, J.F. Huang, D.M. Liang, and Y.F. Tang, Numerical solution of fractional diffusion wave equation based on fractional multistep method, Applied Mathematical Modelling, 38 (2014), pp. 3652-3661.

[35] F.H. Zeng, Second-order stable finite difference schemes for the time-fractional diffusion-wave equation, Journal of Scientific Computing, 65 (2015), pp. 411-430.

\section{Appendix}

Proof of Lemma 2.2: We first consider the case for $2 \leq j \leq k-1$, by Taylor expansion, we have,

$$
f_{j}=f_{j-\frac{1}{2}}+\frac{1}{2} \Delta t f_{j-\frac{1}{2}}^{\prime}+\frac{1}{2 !} \frac{\Delta t^{2}}{4} f_{j-\frac{1}{2}}^{\prime \prime}+\frac{1}{3 !} \frac{\Delta t^{3}}{8} f_{j-\frac{1}{2}}^{\prime \prime \prime}+\frac{1}{4 !} \frac{\Delta t^{4}}{16} f_{j-\frac{1}{2}}^{(4)}+O\left(\Delta t^{5}\right),
$$


and

$$
f_{j-1}=f_{j-\frac{1}{2}}-\frac{1}{2} \Delta t f_{j-\frac{1}{2}}^{\prime}+\frac{1}{2 !} \frac{\Delta t^{2}}{4} f_{j-\frac{1}{2}}^{\prime \prime}-\frac{1}{3 !} \frac{\Delta t^{3}}{8} f_{j-\frac{1}{2}}^{\prime \prime \prime}+\frac{1}{4 !} \frac{\Delta t^{4}}{16} f_{j-\frac{1}{2}}^{(4)}+O\left(\Delta t^{5}\right) .
$$

Subtracting (57) from (56), we get

$$
f_{j}-f_{j-1}=\Delta t f_{j-\frac{1}{2}}^{\prime}+\frac{\Delta t^{3}}{24} f_{j-\frac{1}{2}}^{\prime \prime \prime}+O\left(\Delta t^{5}\right),
$$

which implies that

In addition, we have

$$
f_{j-\frac{1}{2}}^{\prime}=\frac{f_{j}-f_{j-1}}{\Delta t}-\frac{\Delta t^{2}}{24} f_{j-\frac{1}{2}}^{\prime \prime \prime}+O\left(\Delta t^{4}\right)
$$

$$
f_{j}^{\prime}=f_{j-\frac{1}{2}}^{\prime}+\frac{1}{2} \Delta t f_{j-\frac{1}{2}}^{\prime \prime}+\frac{1}{2 !} \frac{\Delta t^{2}}{4} f_{j-\frac{1}{2}}^{\prime \prime \prime}+\frac{1}{3 !} \frac{\Delta t^{3}}{8} f_{j-\frac{1}{2}}^{(4)}+\frac{1}{4 !} \frac{\Delta t^{4}}{16} f_{j-\frac{1}{2}}^{(5)}+O\left(\Delta t^{5}\right),
$$

and

$$
f_{j-1}^{\prime}=f_{j-\frac{1}{2}}^{\prime}-\frac{1}{2} \Delta t f_{j-\frac{1}{2}}^{\prime \prime}+\frac{1}{2 !} \frac{\Delta t^{2}}{4} f_{j-\frac{1}{2}}^{\prime \prime \prime}-\frac{1}{3 !} \frac{\Delta t^{3}}{8} f_{j-\frac{1}{2}}^{(4)}+\frac{1}{4 !} \frac{\Delta t^{4}}{16} f_{j-\frac{1}{2}}^{(5)}+O\left(\Delta t^{5}\right) .
$$

By (59) and (60), we get

$$
f_{j}^{\prime}+f_{j-1}^{\prime}=2 f_{j-\frac{1}{2}}^{\prime}+\frac{\Delta t^{2}}{4} f_{j-\frac{1}{2}}^{\prime \prime \prime}+O\left(\Delta t^{4}\right)
$$

Substituting (58) into the above equation implies that

$$
\frac{f_{j}^{\prime}+f_{j-1}^{\prime}}{2}=f_{j-\frac{1}{2}}^{\prime}+\frac{\Delta t^{2}}{8} f_{j-\frac{1}{2}}^{\prime \prime \prime}+O\left(\Delta t^{4}\right)=\frac{f_{j}-f_{j-1}}{\Delta t}+\frac{\Delta t^{2}}{12} f_{j-\frac{1}{2}}^{\prime \prime \prime}+O\left(\Delta t^{4}\right) .
$$

Noting that

$$
f_{j+1}=f_{j-\frac{1}{2}}+\frac{3}{2} \Delta t f_{j-\frac{1}{2}}^{\prime}+\frac{1}{2 !} \frac{9 \Delta t^{2}}{4} f_{j-\frac{1}{2}}^{\prime \prime}+\frac{1}{3 !} \frac{27 \Delta t^{3}}{8} f_{j-\frac{1}{2}}^{\prime \prime \prime}+\frac{1}{4 !} \frac{81 \Delta t^{4}}{16} f_{j-\frac{1}{2}}^{(4)}+O\left(\Delta t^{5}\right),
$$

and

$$
f_{j-2}=f_{j-\frac{1}{2}}-\frac{3}{2} \Delta t f_{j-\frac{1}{2}}^{\prime}+\frac{1}{2 !} \frac{9 \Delta t^{2}}{4} f_{j-\frac{1}{2}}^{\prime \prime}-\frac{1}{3 !} \frac{27 \Delta t^{3}}{8} f_{j-\frac{1}{2}}^{\prime \prime \prime}+\frac{1}{4 !} \frac{81 \Delta t^{4}}{16} f_{j-\frac{1}{2}}^{(4)}+O\left(\Delta t^{5}\right) .
$$

we have, by (56), (57), (62), (63),

$$
\begin{aligned}
& a f_{j+1}+b f_{j}+c f_{j-1}+d f_{j-2} \\
= & (a+b+c+d) f_{j-\frac{1}{2}}+\left(\frac{3}{2} a+\frac{1}{2} b-\frac{1}{2} c-\frac{3}{2} d\right) \Delta t f_{j-\frac{1}{2}}^{\prime} \\
& +\left(\frac{9}{4} a+\frac{1}{4} b+\frac{1}{4} c+\frac{9}{4} d\right) \frac{\Delta t^{2}}{2 !} f_{j-\frac{1}{2}}^{\prime \prime}+\left(\frac{27}{8} a+\frac{1}{8} b-\frac{1}{8} c-\frac{27}{8} d\right) \frac{\Delta t^{3}}{3 !} f_{j-\frac{1}{2}}^{\prime \prime \prime} \\
& +\left(\frac{81}{16} a+\frac{1}{16} b+\frac{1}{16} c+\frac{81}{16} d\right) \frac{\Delta t^{4}}{4 !} f_{j-\frac{1}{2}}^{(4)}+O\left(\Delta t^{5}\right) .
\end{aligned}
$$

To make the right side of (64) have the third order accuracy, we assume

$$
\begin{aligned}
& a+b+c+d=0, \\
& \frac{3}{2} a+\frac{1}{2} b-\frac{1}{2} c-\frac{3}{2} d=0, \\
& \frac{9}{4} a+\frac{1}{4} b+\frac{1}{4} c+\frac{9}{4} d=0, \\
& \frac{27}{8} a+\frac{1}{8} b-\frac{1}{8} c-\frac{27}{8} d=1,
\end{aligned}
$$

which implies that

$$
a=1 / 6, b=-1 / 2, c=1 / 2, d=-1 / 6 .
$$

Thus we have, by (64),

$$
\frac{1}{6} f_{j+1}-\frac{1}{2} f_{j}+\frac{1}{2} f_{j-1}-\frac{1}{6} f_{j-2}=\frac{\Delta t^{3}}{3 !} f_{j-\frac{1}{2}}^{\prime \prime \prime}+O\left(\Delta t^{5}\right) .
$$


Substituting (65) into (61), we obtain

$$
\frac{f_{j}^{\prime}+f_{j-1}^{\prime}}{2}=\frac{1}{12}\left(\delta_{t} f_{j+\frac{1}{2}}+10 \delta_{t} f_{j-\frac{1}{2}}+\delta_{t} f_{j-\frac{3}{2}}\right)+O\left(\Delta t^{4}\right),
$$

which shows (22).

Next we consider (21), for $j=1$, we have, by Taylor expansion

$$
f_{\frac{1}{2}}=f_{0}+\frac{1}{2} \Delta t f_{0}^{\prime}+\frac{1}{8} \Delta t^{2} f_{0}^{\prime \prime}+\frac{1}{48} \Delta t^{3} f_{0}^{\prime \prime \prime}+O\left(\Delta t^{4}\right),
$$

and

$$
f_{\frac{1}{2}}=f_{1}-\frac{1}{2} \Delta t f_{1}^{\prime}+\frac{1}{8} \Delta t^{2} f_{1}^{\prime \prime}-\frac{1}{48} \Delta t^{3} f_{1}^{\prime \prime \prime}+O\left(\Delta t^{4}\right) .
$$

Subtracting (67) from (68), we get

$$
\frac{f_{0}^{\prime}+f_{1}^{\prime}}{2}=\delta_{t} f_{\frac{1}{2}}+\frac{\Delta t}{8}\left(f_{1}^{\prime \prime}-f_{0}^{\prime \prime}\right)-\frac{\Delta t^{2}}{48}\left(f_{1}^{\prime \prime \prime}+f_{0}^{\prime \prime \prime}\right)+O\left(\Delta t^{3}\right),
$$

where

$$
f_{1}^{\prime \prime}-f_{0}^{\prime \prime}=\Delta t f_{0}^{\prime \prime \prime}+\frac{\Delta t^{2}}{2} f_{0}^{(4)}+O\left(\Delta t^{3}\right) .
$$

Hence we have, by (69) and noting that $f_{1}^{\prime \prime \prime}-f_{0}^{\prime \prime \prime}=\Delta t f_{0}^{(4)}+O\left(\Delta t^{2}\right)$,

$$
\begin{aligned}
\frac{f_{0}^{\prime}+f_{1}^{\prime}}{2} & =\delta_{t} f_{\frac{1}{2}}+\frac{\Delta t^{2}}{48}\left(f_{0}^{\prime \prime \prime}-f_{1}^{\prime \prime \prime}\right)+\frac{1}{12} \Delta t^{2} f_{0}^{\prime \prime \prime}+O\left(\Delta t^{3}\right) \\
& =\delta_{t} f_{\frac{1}{2}}+\frac{\Delta t^{2}}{12} f_{0}^{\prime \prime \prime}+O\left(\Delta t^{3}\right) .
\end{aligned}
$$

Noting that

$$
\begin{aligned}
& f_{1}=f_{0}+\Delta t f_{0}^{\prime}+\frac{\Delta t^{2}}{2} f_{0}^{\prime \prime}+\frac{\Delta t^{3}}{6} f_{0}^{\prime \prime \prime}+O\left(\Delta t^{4}\right), \\
& f_{2}=f_{0}+2 \Delta t f_{0}^{\prime}+2 \Delta t^{2} f_{0}^{\prime \prime}+\frac{4 \Delta t^{3}}{3} f_{0}^{\prime \prime \prime}+O\left(\Delta t^{4}\right), \\
& f_{3}=f_{0}+3 \Delta t f_{0}^{\prime}+\frac{9}{2} \Delta t^{2} f_{0}^{\prime \prime}+\frac{9 \Delta t^{3}}{2} f_{0}^{\prime \prime \prime}+O\left(\Delta t^{4}\right),
\end{aligned}
$$

we get

$$
3 f_{1}-3 f_{2}+f_{3}=f_{0}+\Delta t^{3} f_{0}^{\prime \prime \prime}+O\left(\Delta t^{4}\right)
$$

By (70) and (74), we have

$$
\frac{f_{1}^{\prime}+f_{0}^{\prime}}{2}=\frac{1}{12}\left(13 \delta_{t} f_{\frac{1}{2}}-2 \delta_{t} f_{\frac{3}{2}}+\delta_{t} f_{\frac{5}{2}}\right)+O\left(\Delta t^{3}\right)
$$

which shows (22).

Finally we consider (23), for $j=k$, we have

$$
\frac{f_{k}^{\prime}+f_{k-1}^{\prime}}{2}=\frac{1}{12}\left(13 \delta_{t} f_{k-\frac{1}{2}}-2 \delta_{t} f_{k-\frac{3}{2}}+\delta_{t} f_{k-\frac{5}{2}}\right)+O\left(\Delta t^{3}\right) .
$$

Together these estimates complete the proof of Lemma 2.2 . 